Revue d'archéologie préhistorique

\title{
Contribution des méthodes magnétiques à l'étude
} du remplissage du site préhistorique de l'Abri Pataud (Les Eyzies-de-Tayac, Dordogne, France)

Magnetic parameters contribution to the study of Abri pataud prehistoric site

Les Eyzies-de-Tayac (Dordogne, France)

Abderrezak Djerrab, lan Hedley, Roland Nespoulet et Laurent Chiotti

\section{(2) OpenEdition}

Journals

Édition électronique

URL : https://journals.openedition.org/paleo/644

DOI : $10.4000 /$ paleo.644

ISSN : 2101-0420

Éditeur

SAMRA

Édition imprimée

Date de publication : 30 décembre 2007

Pagination : 281-302

ISSN : $1145-3370$

\section{Référence électronique}

Abderrezak Djerrab, lan Hedley, Roland Nespoulet et Laurent Chiotti, « Contribution des méthodes magnétiques à l'étude du remplissage du site préhistorique de l'Abri Pataud (Les Eyzies-de-Tayac, Dordogne, France) », PALEO [En ligne], 19 | 2007, mis en ligne le 23 avril 2009, consulté le 26 juin 2021. URL : http://journals.openedition.org/paleo/644 ; DOI : https://doi.org/10.4000/paleo.644

\section{(c) (i) (9)}

PALEO est mis à disposition selon les termes de la licence Creative Commons Attribution - Pas d'Utilisation Commerciale - Pas de Modification 4.0 International. 


\title{
CONTRIBUTION DES MÉTHODES MAGNÉTIQUES A L'ÉTUDE DU REMPLISSAGE DU SITE PRÉHISTORIQUE DE L'ABRI PATAUD (Les Eyzies-de-Tayac, Dordogne, France)
}

\author{
Abderrezak DJERRAB ${ }^{(1)}$, lan HEDLEY(2), \\ Roland NESPOULET ${ }^{(3)}$ et Laurent CHIOTTI ${ }^{(4)}$
}

\begin{abstract}
Résumé : Le site de l'Abri Pataud, daté du Pléistocène supérieur (stade isotopique 3 à 2), est caractérisé par une grande richesse en faune et en industrie et par la succession de plusieurs niveaux d'habitats riches en éléments brûlés et en matériel archéologique. L'étude magnétique a permis de faire la distinction entre deux types d'ensembles magnétostratigraphiques, d'une part les éboulis caractérisés par un faible pourcentage en grains magnétiques et par de faibles valeurs des paramètres magnétiques, et d'autre part des niveaux sablo-limoneux, riches en foyers, et présentant de fortes valeurs magnétiques.

Dans les éboulis, les grains magnétiques sont plutôt grossiers (poly-domaines), essentiellement d'origine détritique (hématite, sidérite et goethite). Au contraire, les niveaux archéologiques présentent des grains magnétiques de taille fine (MD, PMD et SP), résultant de la transformation, sous l'effet de la température des foyers, d'oxydes, d'hydroxydes (goethite) et de carbonates de fer $\left(F e C O_{3}\right)$ en oxydes de fer secondaires de taille fine (magnétite et maghémite).
\end{abstract}

Mots-clés : Pléistocène supérieur, Abri Pataud, Susceptibilité Magnétique, Magnétite, Maghémite, Goethite et Hématite.

Key-words: Upper Pleistocene, Abri Pataud, Magnetic Susceptibility, Magnetite, Maghemite, Goethite and Hematite.

\begin{abstract}
Abridged english version
MAGNETIC PARAMETERS CONTRIBUTION TO THE STUDY OF ABRI PATAUD PREHISTORIC SITE LES EYZIES-DE-TAYAC (DORDOGNE, FRANCE).

Introduction

A magnetic study has been carried out on the sedimentary deposit of the prehistoric site of Abri Pataud, situated in the south east of the French department of Dordogne, on the banks of the Vézère valley at an altitude of around $76 \mathrm{~m}$. This Upper Pleistocene site (dated between around 34000 and $20400 \mathrm{BP}$ ), having revealed human bones and an abundant lithic industry, has been studied by numerous authors since the beginning of the twentieth century. This present investigation complements many other previous studies at this site covering different domains: sedimentology, study of tools, fauna, as well as palynology. The stratigraphy of the site, as well as the dates of the principal layers are presented in table 1, whilst a list of the magnetic parameters used is given in table 2 (along with an explanation of their utility and significance, whenever possible). Two different methods were used; firstly direct measurements in the
\end{abstract}

(1) Centre Universitaire de Tébessa, Institut des Sciences de la Terre, Route de Constantine 12002 Tébessa, Algérie, et UMR 5590 du CNRS CERP de Tautavel, France - djerrab@yahoo.fr

(2) Université de Genève, Département de Minéralogie, 13 rue des Maraîchers, 1205 Genève, Suisse - ian.hedley@bluewin.ch

(3) Département de Préhistoire du Muséum national d'histoire naturelle, UMR 5198, USM 103, Institut de paléontologie humaine, 1 rue René Panhard, 75013 Paris, France - roland.nespoulet@mnhn.fr

(4) Département de Préhistoire du Muséum national d'histoire naturelle, UMR 5198, USM 103, Musée de l'abri Pataud, 20 rue du Moyen-âge, 24620 Les Eyzies-de-Tayac, France - Ichiotti@mnhn.fr 
field, then followed by detailed measurements in the laboratory on selected sediment samples. The volume magnetic susceptibility was measured on exposed sections in the rock shelter using a portable magnetic susceptibility meter (Bartington MS2F) and the results represented on coloured contour plots in order to distinguish between different layers and to reveal the presence of hearths. This parameter is proportional to the concentration of magnetic grains present. Some 90 sediment samples in powder form that were taken from five stratigraphic sections $(69 / 70,70 / 71,72 / 73,74 / 75$ and $76 / 77$, tab. 3 and fig. 2) were subjected to varying magnetic fields and temperatures in order to determine standard magnetic parameters. Two different approaches were used. The magnetic hysteresis data were plotted in a so-called Day plot to give information on the grain size of the magnetic minerals present. Further magnetic treatment included a thermal demagnetisation up to $590^{\circ} \mathrm{C}$ of a saturation magnetisation given successively in three orthogonal directions (Lowrie plot) in order to determine the nature of the magnetic grains present. The principal aim was to identify the different magnetic minerals present and their granulometry, as well as to understand their origin, and to throw light on the climate during their formation. Climate having an influence on the minerals formed. The presence of hearths was also confirmed.

\section{Results}

The magnetic study revealed six layers, listed here from the base to the summit. The principal results are also given in table 4 , whilst figures 4 to 10 present the variations of the different magnetic parameters shown as a function of depth within the sections.

- Layer 1 (72cm thick) Rock-fall debris, light colour, rich in $\mathrm{CaCO}_{3}$, corresponding to base debris. Particularly low magnetic susceptibility. Magnetic grains are few and are of detrital origin (that is resulting from a mechanical desegregation and have not suffered a later geochemical evolution), produced by a cold climate, which is confirmed by palynological studies. The magnetic grains have a high coercive force, principally hematite and probably goethite.

- Layer $2(153 \mathrm{~cm}$ thick) is a sandy-silty layer, dark to light brown in colour, with generally higher susceptibility values, notably at certain places corresponding to hearths. In the debris zones the magnetic grains are the same as in layer 1 , whilst in the archaeological layers the grains are secondary (magnetite, maghemite and hematite) produced by the transformation of primary grains by heating in the hearths. The presence of maghemite in the hearths is in agreement with published studies in the literature. The size of these magnetic grains is variable, although dominated by fine grains. The presence of hearths is also indicated by the colour of the sediments along with visible ash and charcoal.

- Layer $3(130 \mathrm{~cm}$ thick) is a formed of light coloured rock-fall debris of low susceptibility with values close to that of layer 1.

- Layer $4(215 \mathrm{~cm}$ thick) can be divided into several sub-layers (sands, reddish brown sediments, silty layers....). The values of the magnetic parameters are high and the iron oxides are secondary, above all magnetite and maghemite. Several hearths (detected due to their dark brown colour, the presence of ash, and of course high values of the magnetic parameters) and archaeological layers are present. Outside of these zones are debris layers with similar magnetic properties to those of layers 1,3 and 5 .

- Layer 5 ( $45 \mathrm{~cm}$ thick) consists of rock debris of low susceptibility but slightly higher than the values in layers 1 and 3 .

- Layer 6 (up to the summit) can also be split up into sub-layers (rock debris and darker layers containing earth showing signs of pedogenesis). The magnetic values are high; the iron oxides that are essentially secondary and fine grained, are the result of pedogenesis (clearly visible in certain places) and hearths.

\section{Conclusion}

The site of Abri Pataud is characterised by a series of occupation layers (dated from the Aurignacian up till the Solutrean), that are separated by sterile rock-fall debris and deposited during a globally cold climate between 35000 and 20000 years ago (isotopic stages 2 and 3). Hearths are abundant in the occupation layers.

It is important to point out that the sedimentary deposit in this site does not show well limited layers as such, rather a piling- up of blocks, stones and finer sediments separated by the hearths that characterise the archaeological layers. However, the magnetic study of the sediments has revealed several layers, three of these showing high values of the magnetic parameters (layers 2,4 , and 6 ) and three others with much lower values (layers 1,3 , and 5$)$. On the one hand certain layers (2, 4 and 6), which correspond to archaeological layers with hearths, show high values of magnetic parameters and contain secondary iron oxides such as fine grained magnetite and maghemite that are largely produced by combustion in the hearths or during pedogenetic phases (particularly in the uppermost layer). On the other hand, layers 1,3 and 5 are made up of rock-fall debris formed in-situ by the alteration of blocks and stones fallen from the limestone wall of the rock shelter, mixed with aeolian sand. These layers are characterised by weak magnetic values, due to a low concentration of magnetic grains that are mainly of a detrital origin.

\section{INTRODUCTION}

Nous allons, dans cet article, nous intéresser au site de l'Abri Pataud, localisé au sud-est du département de la Dordogne, sur la rive droite de la Vézère, qui traverse la commune des Eyzies-de-Tayac (fig.1). Ce site, connu depuis la fin du XIXe siècle, a été fouillé notamment par $\mathrm{H}$. L. Movius (Movius 1975, 1977) et a fait l'objet, plus récemment, d'une opération archéologique programmée dirigée par deux d'entre nous (Nespoulet et Chiotti 2007a). L'Abri Pataud est un vaste abri-sous-roche qui s'est effondré à plusieurs reprises durant le Pléistocène supérieur.

Son remplissage, formé d'une part de débris calcaires provenant des parois (les plus importants en volume), d'autre part de sédiments éoliens et de débris introduits par l'hom- me, atteint une épaisseur maximale de plus de $9 \mathrm{~m} . \mathrm{H}$. L. Movius y a identifié une succession de 14 niveaux archéologiques principaux, dont certains sont extrêmement riches en matériel archéologique (Nespoulet et Chiotti 2007b). Afin de distinguer les niveaux archéologiques des niveaux considérés comme archéologiquement stériles, $\mathrm{H}$. L. Movius a désigné les premiers par des chiffres (par exemple Level 4) et les seconds par une numérotation intermédiaire (par exemple Eboulis 4/5) (Movius 1977 ; Chiotti et Nespoulet 2007).

La séquence culturelle de l'Abri Pataud comporte neuf niveaux aurignaciens, quatre niveaux gravettiens et un niveau solutréen datant, pour le plus ancien, de -34 000 ans et pour le plus récent de -20000 ans. W. R. Farrand, lors de son étude du remplissage, a proposé un découpa- 

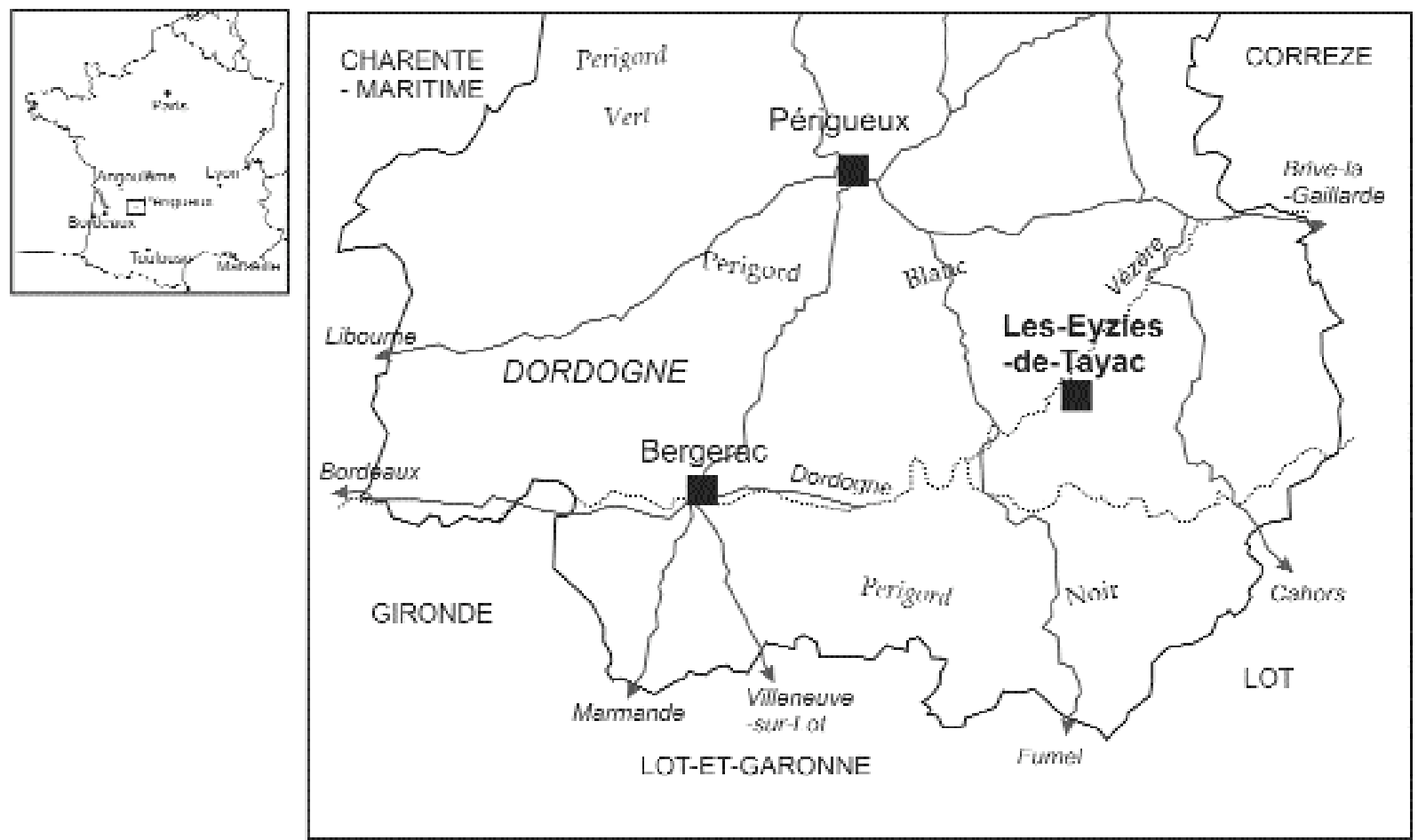

Figure 1 - Situation géographique de l'Abri Pataud (Les Eyzies-de-Tayac, Dordogne).

Figure 1 - Geographical location of the Abri Pataud (Les Eyzies-de-Tayac, Dordogne).

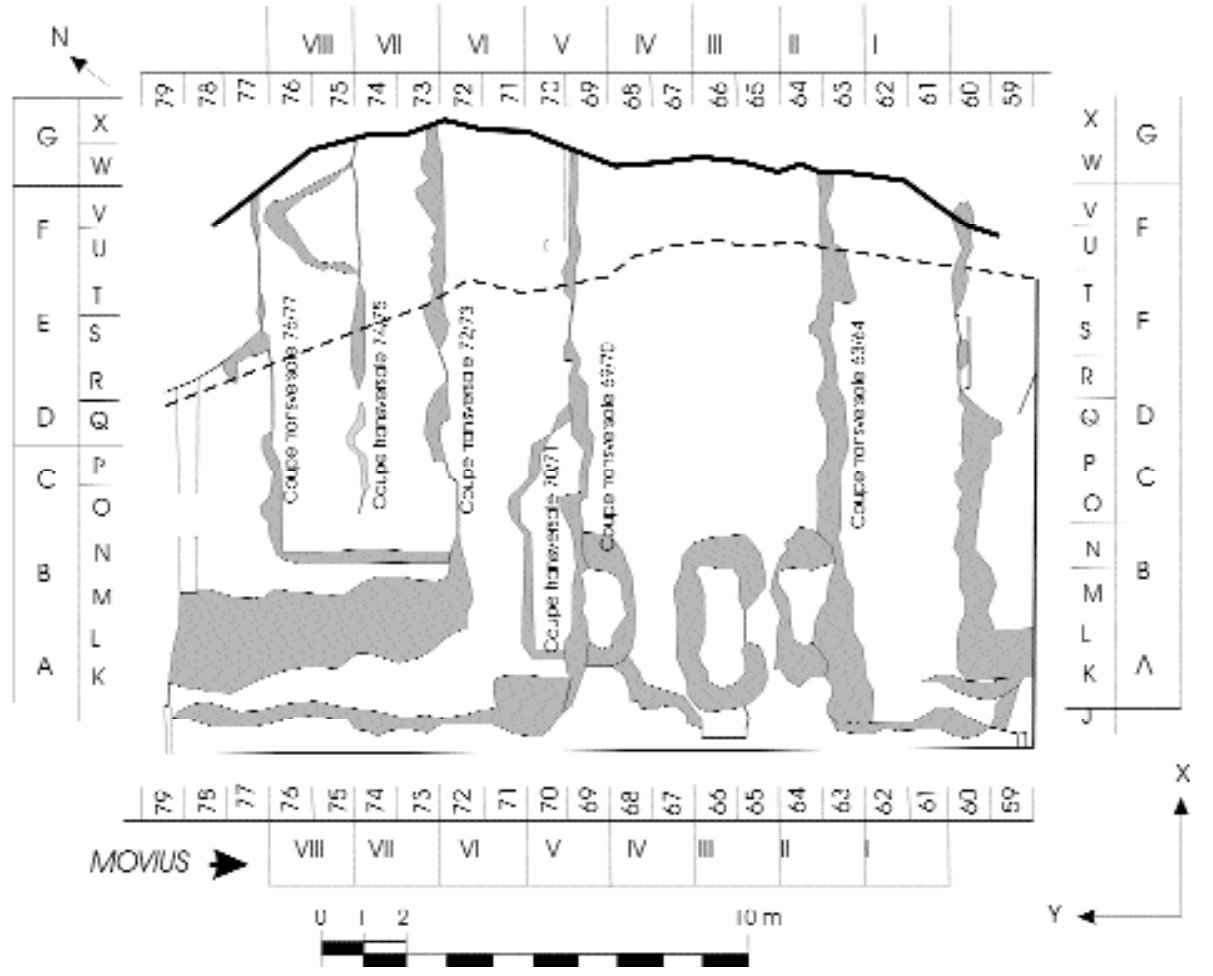

Figure 2 - Plan de l'Abri Pataud (Les Eyzies-de-Tayac, Dordogne).

Figure 2 - Abri Pataud Plan (Les Eyzies-de-Tayac, Dordogne). 


\begin{tabular}{|c|c|c|c|c|}
\hline $\begin{array}{c}\text { Unités } \\
\text { stratigraphiques } \\
\text { (Farrand 1995) }\end{array}$ & $\begin{array}{c}\text { Niveaux } \\
\text { archéologiques } \\
\text { (Movius 1977) } \\
\\
\text { niveun / à } 3 \text { et } \\
\text { éboulis } 0 / 1 \text { à } 3 / 4 \\
\text { Yellow }\end{array}$ & $\begin{array}{l}\text { Epaisscur } \\
\text { (cm) }\end{array}$ & $\begin{array}{l}\text { Description } \\
\text { Sommet de la séquéncé stratigrruphique } \\
\text { éboulis calcuire, de couleur brun clair à } \\
\text { jaune pâle aveo des grruins fins et des } \\
\text { fragments calcuires anguleux (Solutréen } \\
\text { à (iravettien rósent). }\end{array}$ & $\begin{array}{l}\text { Âge } \\
\text { radiocarbone } \\
\text { Nivean } 1: \\
20400 \mathrm{BP} \\
\text { Nivcau } 2: \\
22000 \mathrm{BP} \\
\text { Niveau } 3: \\
24000 \mathrm{BP}\end{array}$ \\
\hline b & éboulis $3 / 4$ & 36 & $\begin{array}{l}\text { limons sableux à sables terreux, de } \\
\text { couleur jaune-rouge à brun foncé } \\
\text { (Gravettien moyen). }\end{array}$ & - \\
\hline $\mathrm{c}$ & niveau 4 & 27 & $\begin{array}{l}\text { éboulis calcaire brun-rouge foncé, à } \\
\text { grains fins et contenant des fragments } \\
\text { calcaires légèrement arrondis (Gravettien } \\
\text { moyen). }\end{array}$ & $\begin{array}{c}\text { Nivcau } 4 ; \\
26000 \text { i } 27000 \\
\text { BP }\end{array}$ \\
\hline d & $\begin{array}{l}\text { cloulis } 4 / 5 \\
\text { niveau } 5\end{array}$ & 27 ì 63 & $\begin{array}{l}\text { éboulis calcaire un peu plus grossier que } \\
\text { dans les unités c ou e, avec des } \\
\text { fragments calcuires plutôt anguleux. } \\
\text { étwoulis calcuire de couleur très variable } \\
\text { (bran foncé, brun-rouge foncé, brun) et } \\
\text { tragments calcaires arrondis (Gravetrien } \\
\text { ancien). }\end{array}$ & $\begin{array}{l}\text { Nivcau } 5: \\
28000 \mathrm{BP}\end{array}$ \\
\hline$f$ & $\begin{array}{c}\text { niveuux } 6 \text { à } l 4 \text { él } \\
\text { éboulis } 5 / 6 \dot{a} \\
1.3 / / 4\end{array}$ & 478 & $\begin{array}{l}\text { éboulis calcaire jaune pâle à brun très } \\
\text { clair, à grains grossiers et matrice } \\
\text { sableuse, contenant de nombreux bloces } \\
\text { calcaircs (Aurignacien ancien et ćvoluć). }\end{array}$ & $\begin{array}{l}\text { Niveaux } 6 \text { à } 14 \text { : } \\
30000 \text { à } 340001 \\
\text { BP }\end{array}$ \\
\hline
\end{tabular}

Tableau 1 - Stratigraphie de l'Abri Pataud.

Ce tableau ne détaille pas nos propres résultats, mais les études faites antérieurement :

- étude sédimentologique de Farrand (1995) (niveaux a à f),

- stratigraphique culturelle de Movius (1977) (niveaux 1 à 14), Nespoulet, Chiotti (2007a),

- datations moyennes obtenues principalement à partir des datations au ${ }^{14} \mathrm{C}$ réalisées par le laboratoire de Groningen

(Pays-Bas) Vogel et Waterbolk 1963, 1967, 1972 ; Movius 1963, 1971 et à partir d'autres datations au ${ }^{14} \mathrm{C}$ basée sur le procédé ASM réalisées par le laboratoire d'Oxford, (Royaume-Uni) (Bricker et Mellars 1987)

Table 1 - Stratigraphy of Abri Pataud. 


\begin{tabular}{|c|c|}
\hline Symbole & Signilication \\
\hline $\mathrm{K}_{\mathrm{bff}} \mathrm{et} \mathrm{K}_{\mathrm{hff}}$ & $\begin{array}{l}\text { Susceptibilité magnétique volumique à basse lrequence }(0,46 \mathrm{kll} / \% \text { ) et à haute } \\
\text { fréquence }(4,6 \mathrm{kIl} \text { ). Sans dimension. (Mooncy et al. } 2002)\end{array}$ \\
\hline$\chi_{\mathrm{br}}$ et $\chi_{\mathrm{bl}}$ & $\begin{array}{l}\text { Susceptibilité magnétique massique ả basse lréquence et haute fréquence : } \\
\qquad \chi_{\mathrm{bl} / \mathrm{hl}}=\left(\mathrm{K}_{\mathrm{bl} / \mathrm{hr}} \nu\right) / p\left(\mathrm{~m}^{3} \cdot \mathrm{kg}^{-1}\right) \\
\text { avec } v \text { volume et } p \text { masse de l'échantillon. }\end{array}$ \\
\hline$\overline{\chi_{\text {Iij }}}$ & $\begin{array}{l}\text { Dépendanee en fréquence de la suseeptibilité magnétique: } \\
\qquad \chi_{\mathrm{fa}}=\left(\left(\chi_{\mathrm{bl}}-\chi_{\mathrm{bl}}\right) \chi_{\mathrm{bl}}\right) \times 100 \% \text { (Maher } 1986 \text { et } 1988 \text {; Mullins 1977) } \\
\text { Utilisée pour cstimer le pourcentage des grains magnétiques de taille SP }\end{array}$ \\
\hline$T_{\mathrm{c}}$ & $\begin{array}{l}\text { Tempéralure de Curie : au-dessus de laquelle tout corps perd ses propriélés } \\
\text { magnéticues. }\end{array}$ \\
\hline MD & MonoDomaine : Grains de petite taille \\
\hline SP & SuperParamagnélique : Grains de laille très line (quelques nanomètres) \\
\hline PD & $\begin{array}{l}\text { PolyDomaine : Grains de plus grande taille. La limile de laille avec les gr. MD } \\
\text { cst variable (selon le type de matériau et sa forme notamment). }\end{array}$ \\
\hline PMD & Pscudo-MonoDomaine \\
\hline $\mathrm{K}_{\mathrm{n} \mathrm{m}}$ & Susceptibilité anhystérétique \\
\hline $\mathrm{K}_{\text {fermo }}$ & 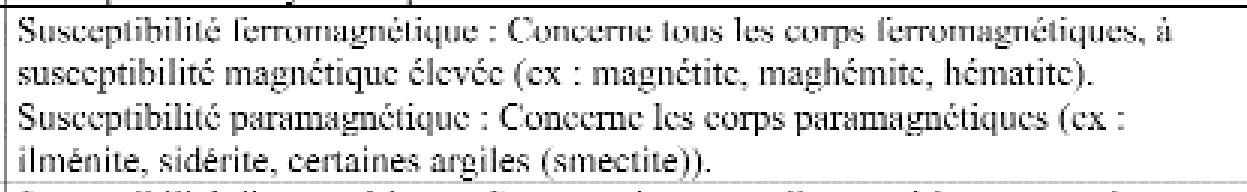 \\
\hline $\mathrm{K}_{\mathrm{d} u}$ & $\begin{array}{l}\text { Susceptibilité diamagnćtique : Conccrne les corps diamagnétiques, constituant en } \\
\text { général la malrice (quarl\%, calcile, tau,...) }\end{array}$ \\
\hline$A R I_{S}$ & $\begin{array}{l}\text { Aimantation Rémanente Isotherme de saturation ( } \mathrm{cn} \Lambda . \mathrm{m}^{2} \cdot \mathrm{kg}^{-1} \text { si massique ou en } \\
\mathrm{N} / \mathrm{m} \text { si volumique) }\end{array}$ \\
\hline$A R I$ & Aimantation Rémanente Isotherme. \\
\hline $\begin{array}{l}\text { ARI- } \\
100 / \text { ARIs }\end{array}$ & Rapport de l'ARI à $100 \mathrm{mT}$ el de l'ARI s (Bloemendal el al. 1992) \\
\hline$\Lambda \mathrm{R} \Lambda$ & $\begin{array}{l}\text { Aimantation Rémanerıle Anhystérétique (en } \mathrm{A}, \mathrm{m}^{2}{ }^{2} \mathrm{~kg}^{-1} \text { si massique ou } \mathrm{cn} N / \mathrm{m} \text { si } \\
\text { volumique) }\end{array}$ \\
\hline $\begin{array}{l}\mathrm{H} \\
\mathrm{Hc}\end{array}$ & $\begin{array}{l}\text { Champ magnétique appliqué } \\
\text { Champ cocreitif de saturation' }\end{array}$ \\
\hline Her & Champ cocreitif remancnt \\
\hline $\begin{array}{l}\text { Ms } \\
\text { Mrs }\end{array}$ & $\begin{array}{l}\text { Aimantalion à saluration } \\
\text { Aimantalion rémanente sponlanée }{ }^{1}\end{array}$ \\
\hline
\end{tabular}

Tableau 2 - Définition des paramètres magnétiques utilisés.

1 - Paramètres définis grâce au cycle d'hystérésis : les variations des rapports Mrs/Ms et Hcr/Hc sont utilisées pour déterminer la microstructure, notamment la forme et la taille des grains (Collombat 1993).

Table 2 - Magnetic parameters definition. 
ge en six unités stratigraphiques principales (Farrand 1975, 1995) (tab. 1).

Notre étude, qui vient en complément de nombreuses autres études antérieures (chronologiques, sédimentologiques, palynologiques ou archéologiques), ne peut en être dissociée. En effet, elle ne peut être vraiment utile que si elle est associée ou comparée à d'autres approches. Elle permet alors de préciser la stratigraphie (les méthodes magnétiques permettent de révéler des variations, notamment dans le mode de dépôt, qui ne sont pas forcément évidentes à l'œil nu), de proposer une interprétation paléoclimatique (une comparaison avec des études faunistiques ou palynologiques s'avère alors utile) et de détecter la présence de foyers (parfois confondus avec des zones riches en matière organique) (Thompson et al. 1975 ; Dearing et Flower 1982 ; Rummery 1983 ; Zhou et al. 1990 ; Maher et Thompson 1992).

Par le biais de l'utilisation de différents paramètres magnétiques, dont nous détaillons la signification dans le tableau 2 (dans la mesure du possible, car nous ne pouvons pas donner ici la signification de toutes les notions physiques évoquées), et après avoir décrit la méthodologie utilisée, nous allons nous efforcer d'apporter une contribution à l'étude de ce site préhistorique de référence.

\section{MÉTHODOLOGIE}

Deux types de méthodes ont été employés : des méthodes de terrain et des méthodes de laboratoire.

- Les analyses de terrain consistent en la mesure de la susceptibilité magnétique volumique $(\mathrm{k})$, à l'aide d'un susceptibilimètre portatif (type Bartington MS2 (fréquence $958 \mathrm{~Hz}$ ) connecté à une sonde de type MS2F). Ces mesures, très fastidieuses (en effet, une mesure doit impérativement être réalisée au plus tous les cinq centimètres, parfois moins en cas de variations notables de l'apparence des sédiments), permettent en général d'obtenir de très bons résultats quant à la différentiation des différents niveaux stratigraphiques ou pour le repérage de sédiments contenant de nombreux éléments chauffés. La susceptibilité magnétique volumique peut être utilisée en première approche pour estimer la concentration en grains magnétiques dans les sédiments (magnétite, maghémite, etc.) (Liu et al. 2004a). Une illustration parlante en est fournie sur la figure 3 .

En outre, pour chaque niveau de prélèvement, une description précise a été réalisée (texture, couleur approximative ou autres caractéristiques utiles).

- Pour effectuer les analyses en laboratoire, de loin les plus conséquentes, 90 échantillons ont été prélevés sur toute la hauteur du remplissage, sur les cinq coupes stratigraphiques transversales suivantes : 69/70, 70/71, 72/73, $74 / 75$ et $76 / 77$ (tab. 3 et fig. 2 ).

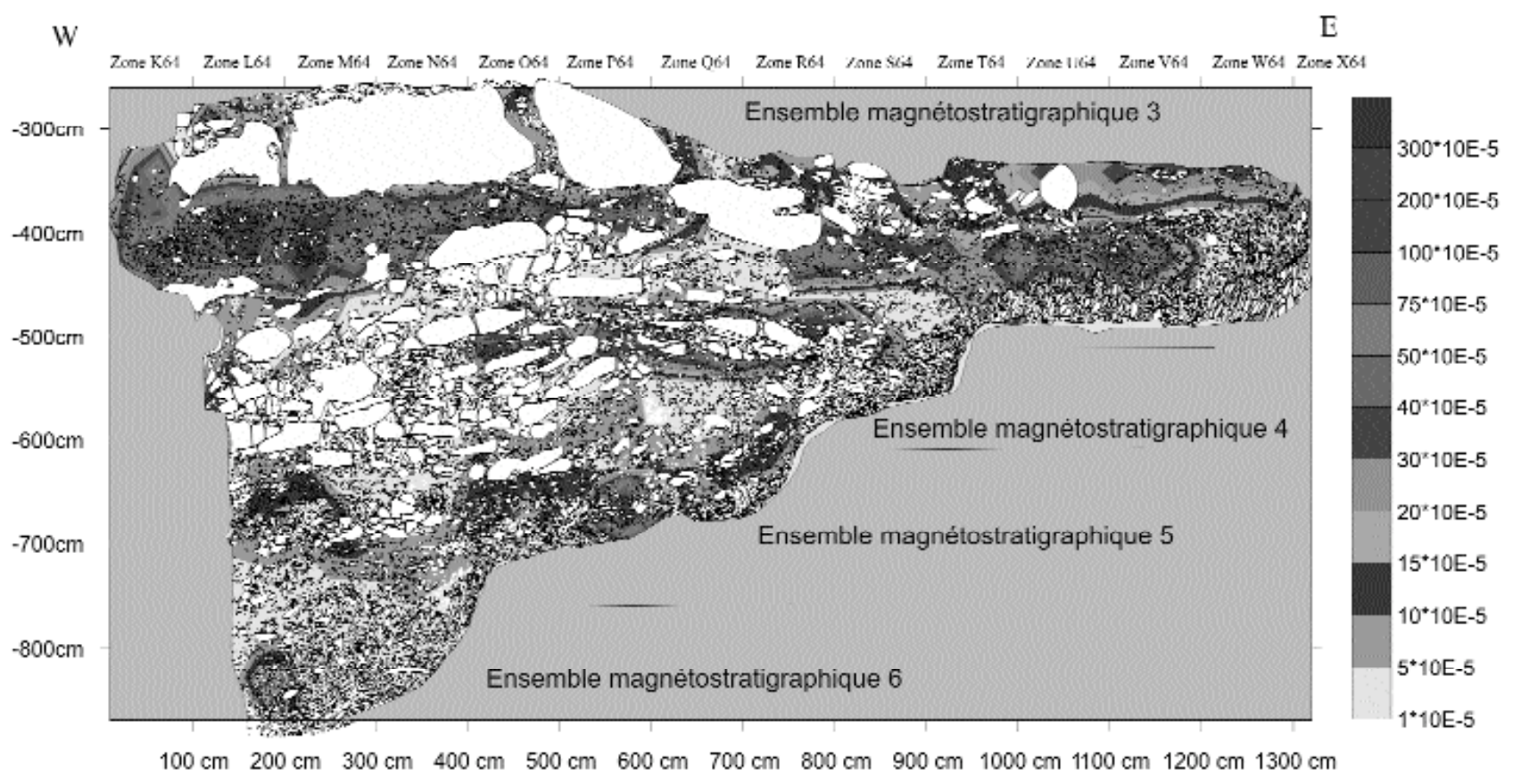

Figure 3 - Représentation graphique de la susceptibilité magnétique volumique ( $k^{\star} 10 E-5$ S.l) sur la coupe stratigraphique transversale 63/64 de l'Abri Pataud.

Figure 3 - Graphical representation of the bulk magnetic susceptibility on the transversal stratigraphic section 63/64 of the Abri Pataud. 


\begin{tabular}{|c|c|c|c|c|c|}
\hline $\begin{array}{c}\text { Numéro } \\
\text { d'échantillon }\end{array}$ & $\begin{array}{l}\text { Profondeur } \\
\mathbf{Z} \text { (en cm) }\end{array}$ & Zone & $\begin{array}{l}\text { Niveau } \\
\text { archéologique } \\
\text { (Movius) }\end{array}$ & attribution culturelle & $\begin{array}{c}\text { Ensemble } \\
\text { magnéto- } \\
\text { stratigraphique }\end{array}$ \\
\hline \multicolumn{6}{|c|}{ Conpe stratigraphique transversale $76 / 77$} \\
\hline AP-1 & -75 & P77 & cboulis $0 / 1$ & & EMS 6 \\
\hline $\mathrm{AP}-2$ & -85 & P77 & éboulis $0 / 1$ & & LMS 6 \\
\hline$\Lambda \mathrm{I}^{2}-3$ & -90 & 177 & bubulis $0 / 1$ & & LMS 6 \\
\hline $\mathrm{AP}-1$ & -9.5 & $\mathrm{P} 77$ & Shoulis $0 / 1$ & & F.MS 6 \\
\hline AP-5 & -105 & P77 & cboulis $0 / 1$ & & EMS 6 \\
\hline AP-G & -115 & P77 & Éboulis $0 / 1$ & & EMS 6 \\
\hline AP-7 & -125 & P77 & niveau 1 & Solutrien (infërieur?) & LMS 6 \\
\hline $\mathrm{Al}^{3}-8$ & -135 & 177 & éboulis $1 / 2$ & & LiMS 6 \\
\hline AP-9 & -145 & $\mathrm{P} 77$ & choulis $1 / 2$ & & F.MS 6 \\
\hline $\mathrm{AP}-10$ & -155 & $\mathrm{P} 77$ & Shoulis $1 / 2$ & & FMS 6 \\
\hline \multicolumn{6}{|c|}{ Coupe stratigraphique transversale $74 / 75$} \\
\hline AP-11 & -175 & $\$ 75$ & éboulis $1 / 2$ & & LMS 6 \\
\hline$\Delta I^{2}-12$ & -185 & $\mathrm{~S} 75$ & éboulis $1 / 2$ & & LMS 6 \\
\hline$A P-13$ & -190 & $\mathrm{~S} 75$ & Shoulis $1 / 2$ & & F.MS 6 \\
\hline $\mathrm{AP}-14$ & -210 & S75 & nivcas 2 & Gravetticn final & F.MS 6 \\
\hline AP-15 & -225 & $\$ 75$ & nivcau 2 & Gravettion final & EMS 6 \\
\hline$A P-16$ & -234 & $\mathrm{~S} 75$ & niveau 2 & Gravettien linal & LMS 6 \\
\hline \multicolumn{6}{|c|}{ Coupe: strutigruphique transwersale $72 / 73$} \\
\hline$A P-17$ & -240 & $\mathrm{~T} 73$ & choulis $2 / 3$ & & FMS 5 \\
\hline$A P-18$ & -250 & T73 & Shoulis $2 / 3$ & & FMS 5 \\
\hline AP-19 & $-2(x)$ & T73 & Eboulis $2 / 3$ & & EMS 5 \\
\hline$A P-20$ & -270 & 173 & éboulis $2 / 3$ & & LMS 5 \\
\hline$A \mathrm{P}-2.1$ & -285 & $\mathrm{~T} 73$ & éboulis $2 / 3$ & & T:MS 4 \\
\hline $\mathrm{AP}-22$ & -295 & $\mathrm{~T} 73$ & choulis $2 / 3$ & & FMS 4 \\
\hline $\mathrm{AP}-23$ & -305 & $\mathrm{~T} 73$ & Choulis $2 / 3$ & & FMS 4 \\
\hline AP-24 & -315 & T73 & ćboulis $2 / 3$ & & EMS 4 \\
\hline$\Lambda P-25$ & -320 & 173 & éboulis $2 / 3$ & & LMS 4 \\
\hline $\mathrm{AP}^{2}-26$ & -330 & $\mathrm{~T} 73$ & éboulis $7 / 3$ & & T:MS 4 \\
\hline $\mathrm{AP}-27$ & -3.35 & $\mathrm{~T} 73$ & choulis $2 / 3$ & & F.MS 4 \\
\hline$A P-28$ & -345 & V73 & choulis $2 / 3$ & & FMS 1 \\
\hline AP-29) & -355 & V73 & cboulis $2 / 3$ & & EMS 4 \\
\hline$\Lambda P-30$ & -365 & V73 & niveau 3 & Graveltien rècenl & LMS 4 \\
\hline$A P^{2}-31$ & -370 & $\sqrt{ } 73$ & nivesu 3 & Gravellien récenl & F:MS 4 \\
\hline $\mathrm{AP}^{2}-3 \%$ & -380 & V73 & nivesu 3 & Giravellien réx:enl & T:MS 4 \\
\hline $\mathrm{AP}-33$ & -390 & V73 & nivcain 3 & Gravettien récent & F.MS 1 \\
\hline AP-34 & $-4(0)$ & $\sqrt{73}$ & nivcau 3 & Gravettien récent & EMS 4 \\
\hline \multicolumn{6}{|c|}{ Coupe stratigraphique transversale $70 / 71$} \\
\hline $\mathrm{AP}-35$ & -350 & 071 & éboulis 3/4 & & T:MS 4 \\
\hline $\mathrm{AP}-36$ & -355 & 071 & éboulis 3/4 & & F:MS 4 \\
\hline $\mathrm{AP}-37$ & -360 & $O 71$ & choulis $3 / 4$ & & FMS 1 \\
\hline$A P-38$ & $-36,5$ & 071 & sboulis 3/4 & & EMS 4 \\
\hline$\Lambda P-39$ & -370 & 071 & éboulis $3 / 4$ & & LMS 4 \\
\hline $\mathrm{AP}-40$ & -375 & 071 & nivesan 4 & Giravetlien moyen & T:MS 4 \\
\hline$A P^{-4 I}$ & -385 & 071 & nivesu 4 & Grisvetlien moyen & T:MS 4 \\
\hline$A P-12$ & -395 & $\mathrm{N7I}$ & nivcain 1 & Gravetticn moyen & F.MS 4 \\
\hline AP-43 & -4() 5 & $\mathrm{~N} 71$ & nivcau 4 & (iravetticn moyen & EMS 4 \\
\hline$\Lambda P-44$ & -410 & N71 & niveau 4 & Graveltien moyen & LMS 4 \\
\hline
\end{tabular}




\begin{tabular}{|c|c|c|c|c|c|}
\hline \multicolumn{6}{|c|}{ Conpe stratigraphique transversale $69 / 70$} \\
\hline $\mathrm{AP}-45$ & -435 & $\mathrm{M} 70$ & niveau 4 & Gruvettien moyen & LMS 4 \\
\hline$A P-46$ & -440 & M70 & niveau 4 & Gruvettien moyen & LMS 4 \\
\hline$\Delta \mathrm{P}^{2}-47$ & -450 & $\mathrm{M} 70$ & nivesu 5 & Gravellien ancien & LiMS 4 \\
\hline $\mathrm{AP}-48$ & -455 & $\mathrm{M} 70$ & nivcias 5 & Graveuien ancien & F.MS A \\
\hline AP-49 & -465 & $\mathrm{M} 70$ & nivcau 5 & Gravetticn ancicn & EMS 4 \\
\hline AP-50 & -470 & M70 & niveau 5 & Gruvettien uncien & LMS 4 \\
\hline AP-51 & -475 & M70 & niveau 5 & Gruvettien uncien & LMS 4 \\
\hline $\mathrm{AP}-52$ & -485 & M70 & nivesu 5 & Gravellien ancien & LiMS 4 \\
\hline $\mathrm{AP}-5.3$ & -192 & $\mathrm{M} 70$ & nivcian 5 & Gravellien ancien & F.MS A \\
\hline AP-54 & -498 & $\mathrm{M} 70$ & éboulis $5 / 7$ & & EMS 4 \\
\hline AP-55 & -480 & $\mathrm{M} 70$ & éboulis $5 / 7$ & & LMS 4 \\
\hline AP-56 & -490 & $M 70$ & éboulis $5 / 7$ & & LMS 4 \\
\hline $\mathrm{NP}^{2}-57$ & -.500 & M70 & éboulis $5 / 7$ & & LMS 3 \\
\hline $\mathrm{AP}-58$ & -522 & M70 & Shoulis $5 / 7$ & & FMS 3 \\
\hline AP-59 & -530 & M70 & ćboulis $5 / 7$ & & EMS 3 \\
\hline$A P-60$ & -538 & M70 & éboulis $5 / 7$ & & LMS 3 \\
\hline$A P-61$ & -543 & 070 & éboulis $5 / 7$ & & LMS 3 \\
\hline $\mathrm{Al}^{2}-62$ & -.548 & 670 & éboulis $5 / 7$ & & LiMS 3 \\
\hline$A P-63$ & -555 & 070 & choulis $5 / 7$ & & F.MS 3 \\
\hline AP- 64 & $-56,3$ & 070 & éboulis $5 / 7$ & & EMS 3 \\
\hline$A P-65$ & -575 & 070 & niveau 7 & Aurngnucien èvoluē & LMS 3 \\
\hline$\Lambda P-66$ & -585 & 070 & éboulis $7 / 8$ & & LMS 3 \\
\hline $\mathrm{AP}^{\mathrm{P}}-6 \mathrm{7}$ & -610 & 070 & éboulis $7 / 8$ & & F:MS 3 \\
\hline$A P-68$ & -620 & 070 & Shoulis $7 / 8$ & & FMS 3 \\
\hline AP- $(y)$ & $-6,30$ & 070 & nivcau 8 & Aurignacion évolué & EMS 2 \\
\hline AP- 70 & $-\{45$ & 070 & nivcau 8 & Aurignacien ćvolué & EMS 2 \\
\hline$\Lambda P-71$ & -655 & 070 & niveau 9 & Aurignacien ancien & LMS 2 \\
\hline $\mathrm{AI}^{\mathrm{P}}-72$. & .665 & 070 & éboulis $9 / 10$ & & FiMS ? \\
\hline $\mathrm{AP}-73$ & -675 & 070 & Ghoulis $10 / 1 \mid$ & & FMS 2 \\
\hline AP-74 & -685 & 070 & ćboulis $10 / 11$ & & EMS 2 \\
\hline AP-75 & $-(6) 5$ & $\mathrm{M} 70$ & nivcau 9) & Aurignacien ancien & EMS 2 \\
\hline$\Lambda P-76$ & -705 & $M 70$ & éboulis 10/11 & & LMS 2 \\
\hline$A \mathrm{P}-77$ & -77.8 & $\mathrm{M} 70$ & nivean II & Aurignascien ancienı & F:MS 2 \\
\hline $\mathrm{AP}-78$ & -735 & $\mathrm{M} 70$ & niveau II & Aurignacien ancien & FMS 2 \\
\hline AP-79) & -742 & $\mathrm{M} 70$ & niveau 11 & Aurignacien ancien & EMS 2 \\
\hline AP- 80 & -750 & $\mathrm{M} 70$ & niveau 11 & Aurignacien ancien & EMS 2 \\
\hline$\Lambda P-81$ & -756 & $\mathrm{M} 70$ & niveau 11 ou 12 ? & Nurignacien ancien & LMS 2 \\
\hline$A \mathrm{P}-82$. & -763 & $\mathrm{M} 70$ & niveatu 12 vo 13 ? & Aurigniscien ancienı & F:MS 2 \\
\hline$A P-83$ & -775 & M70 & nivcau 12 on 13 ? & Aurignacien ancien & FMS 2 \\
\hline AP-84 & $-7 \times 3$ & $\mathrm{M} 70$ & cboulis de hase & & EMS 1 \\
\hline AP- 85 & -790 & M70 & cboulis de hase & & EMS 1 \\
\hline$A P-86$ & -800 & $M 70$ & ëboulis de bease & & LMS 1 \\
\hline $\mathrm{AP}-87$ & -810 & $\mathbf{M} 70$ & Éboulis de base & & T:MS I \\
\hline$A P-88$ & -817 & M70 & Eboulis de hase & & FMS I \\
\hline AP- 89 & -830 & M70 & Eboulis de hase & & EMS 1 \\
\hline AP-SO & -855 & $\mathrm{M} 70$ & Eboulis de hase & & EMS 1 \\
\hline
\end{tabular}

Tableau 3 - Localisation et attribution stratigraphique des échantillons prélevés.

Table 3 - Samples location and stratigraphic attribution. 
Un certain nombre de paramètres, détaillés ci-après (et résumés dans le tableau 2 ), ont pu être déterminés :

- La susceptibilité magnétique massique $(\chi)$, mesurée à basse fréquence $(0,46 \mathrm{kHz})$ et à haute fréquence $(4,65$ $\mathrm{kHz}$ ), permet de calculer la dépendance en fréquence de la susceptibilité magnétique $\left(\chi_{\mathrm{fd}}\right)$ (Maher 1986, 1988 ; Mullins 1977) (appareil MS2 connecté à une sonde MS2B, laboratoire de Tautavel, France).

Au-delà d'un certain diamètre, la susceptibilité magnétique massique, ainsi que la susceptibilité volumique ( $\kappa$ et $\chi_{b f}$ ) (fig. 3 et 4 ), est peu sensible à la variation de la taille des grains de magnétite (Heider et al. 1996) : elle peut donc être utilisée pour estimer la concentration en grains magnétiques dans les sédiments (Liu et al. 2004a).

- L'aimantation rémanente isotherme à saturation (ARIs) a été mesurée en utilisant un électro-aimant, grâce auquel un fort champ magnétique (1 $\mathrm{T}$ ) est appliqué sur l'échantillon suivant une direction déterminée, à la suite de quoi l'ARI $\mathrm{s}_{\mathrm{s}}$ est mesurée à l'aide d'un magnétomètre (de type "spinner", au laboratoire de pétrophysique de l'université de Genève). Finalement, une aimantation rémanente anhystéritique (ARA) est induite en appliquant un faible champ direct de $0,2 \mathrm{mT}$, puis un fort champ altern atif de 0,12 T sur l'échantillon dans le sens inverse de l'aimantation à saturation. L'ARA acquise est ensuite mesurée dans le magnétomètre (Liu et al. 2004b).

Ce dernier paramètre est très influencé par la présence de grains de taille MD et PMD (Hunt et al. 1995), et donc le rapport $A R I_{S} / A R A$ peut être utilisé pour estimer la contribution de chaque type de grains magnétiques (MD, PMD et PD). Dans notre expérience, l'ARI acquise à $100 \mathrm{mT}$ et à $1000 \mathrm{mT}$ caractérise la présence de grains magnétiques de champ coercitif fort, comme l'hématite et la goethite, et les grains de taille MD (Robinson 1986 ; Bloemendal et al. 1992).

Par ailleurs, il est important de noter que certains paramètres peuvent indiquer la présence d'un minéral particur lier. Par exemple, le rapport $A R I_{s} / \chi_{b f}$ est souvent utilisé pour indiquer la présence de pyrrhotite. Dekkers (1988) a observé que la présence de pyrrhotite était corrélée avec des valeurs élevées de ce rapport de même pour l'hématite, la goethite et la greigite (Peters et Dekkers 2003 ; Roberts et al. 1995). De faibles valeurs de ce rapport (inférieures à 4 $\mathrm{kA} / \mathrm{m}$ ) indiquent au contraire la présence de maghémite, de magnétite ou de titanomagnétite (aux propriétés magnétiques douces)

- Le champ coercitif de la rémanence $\left(H_{c r}\right)$, qui est le champ nécessaire pour annuler l'aimantation à saturation, est mesuré de la façon suivante : un champ à palier croissant (à partir de $5 \mathrm{mT}$ ) est appliqué dans le sens inverse de l'aimantation à saturation, puis l'échantillon est placé dans un magnétomètre et l'intensité de l'aimantation est mesurée ;

- enfin, l'aimantation à saturation $\left(\mathbf{M}_{\mathrm{s}}\right)$, l'aimantation rémanente spontanée $\left(\mathbf{M}_{\mathrm{rs}}\right)$, le champ coercitif $\left(\mathbf{H}_{c}\right)$ et le champ coercitif rémanent $\left(\mathbf{H}_{\mathrm{cr}}\right)$ sont définis à l'aide $\mathrm{du}$ cycle d'hystérésis, procédure par laquelle un corps magnétique acquiert une aimantation rémanente. La forme du cycle d'hystérésis ainsi que les valeurs des rapports $M_{r s} / M_{s}$ et $\mathrm{H}_{\mathrm{cr}} / \mathrm{H}_{\mathrm{c}}$ dépendent de la microstructure, principalement de la forme et de la taille des grains magnétiques (Collombat 1993 ; Hartstra 1982). Hcr/Hc augmente avec la taille des particules tandis que $M_{r s} / M_{s}$ montre une évolution inverse (Dekkers 2003). Les mesures de cycle d'hystérésis ont été effectuées au laboratoire du CEREGE à Marseille.

Par ailleurs, quelques échantillons ont également subi la procédure proposée par Lowrie (1990). Tout d'abord, une ARI est appliquée le long de l'axe $Z$ de l'échantillon (champ de 1T), une seconde ARI le long de l'axe $X$ (champ de 100 $\mathrm{mT}$ ) et enfin une troisième suivant l'axe $Y$ (champ de 50 $\mathrm{mT})$. Finalement, les échantillons sont désaimantés thermiquement. Cette procédure permet une bonne séparation des différentes composantes magnétiques et de leurs champs coercitifs (Lowrie 1990 ; Westphal et al. 1998). La valeur du champ coercitif peut donner une indication sur la nature des grains magnétiques (fort champ coercitif pour les grains d'origine détritique " antifemomagnétiques " qui résultent de la désagrégation de la roche mère sous des conditions climatiques rigoureuses (froid et sec) (Heller et Liu 1982), et faible champ coercitif pour les grains de magnétite et de maghémite (etc) formés sous un climat humide et chaud (Deng et al. 2000). Sous un climat froid, la roche calcaire se désagrège sur place pour donner des cailloux gélifs et du sable calcaire. Le calcaire contient souvent de la sidérite $\left(\mathrm{FeCO}_{3}\right.$, qui est une matière paramagnétique) et d'autres minéraux de fort champ coercitif.

Avant d'entamer la présentation des résultats, il est utile d'apporter quelques précisions sur la notion de susceptibilité magnétique. Tout matériel placé sous un champ magnétique devient magnétique. La susceptibilité magnétique est indicatrice de l'intensité de cette magnétisation. Elle est différente de la rémanence des roches (qui est l'aimantation rémanente naturelle fossilisée par les grains magnétiques au moment de leur formation ou de leur mise en place)

La susceptibilité magnétique est généralement considérée comme révélatrice de la concentration en minéraux magnétiques dans les différents types de roches. Elle peut être mesurée facilement et rapidement sur le terrain, et au laboratoire sur de petits échantillons. La plupart des minéraux, et plus principalement ceux qui contiennent du fer, sont susceptibles d'être magnétisés dans un champ magnétique.

Le Borgne $(1955,1964)$ a signalé que la susceptibilité magnétique augmentait dans les foyers par la réduction de I'hématite $\left(\alpha-\mathrm{Fe}_{2} \mathrm{O}_{3}\right)$ en magnétite $\left(\mathrm{Fe}_{3} \mathrm{O}_{4}\right)$ ou en maghémite $\left(\gamma-\mathrm{Fe}_{2} \mathrm{O}_{3}\right)$. La goethite aussi peut se convertir en magnétite pendant la chauffe et en présence de matière organique (bois ou autres végétaux) (Mullins 1977 ; Rummery et al. 1979).

Par ailleurs, il est important de souligner que dans les grottes et abris-sous-roche, dont l'encaissant est une roche calcaire, les oxydes de fer sont en général relative- 
ment peu abondants. Ils peuvent cependant provenir de la dissolution de la paroi calcaire, qui libère du fer à partir de la sidérite $\left(\mathrm{FeCO}_{3}\right)$ ou de la lépidocrocite $(\gamma \mathrm{FeOOH})$.

Ces deux minéraux, qui sont qualifiés de primaires, peuvent subir des transformations. Ainsi, la déshydratation de la lépidocrocite donne naissance, sous l'effet de la chaleur, à de la maghémite, tandis que l'oxydation de la sidérite donne de l'hématite, qui est une forme stable, accompagnée de deux oxydes instables (magnétite $\left(\mathrm{Fe}_{3} \mathrm{O}_{4}\right)$ et de maghémite $\left.\left(\gamma \mathrm{Fe}_{2} \mathrm{O}_{3}\right)\right)$ selon les deux réactions suivantes :

$$
\begin{aligned}
& 2 \mathrm{FeCO}_{3}+1 / 2 \mathrm{O}_{2} \Longleftrightarrow \gamma \mathrm{Fe}_{2} \mathrm{O}_{3}+2 \mathrm{CO}_{2} \\
& 3 \mathrm{FeCOB}+1 / 2 \mathrm{O} 2 \Longleftrightarrow \mathrm{Fe}_{3} \mathrm{O}_{4}+3 \mathrm{CO}_{2}
\end{aligned}
$$

Les deux oxydes obtenus (maghémite et magnétite), très instables, vont subir plusieurs oxydations complexes et être transformés en hématite (Ellwood et al. 1986). La réaction chimique peut durer plus de 1000 ans dans le cas des grains MD de sidérite les plus volumineux, et seulement quelques heures ou quelques jours dans le cas de grains fins.

\section{RÉSULTATS ET INTERPRÉTATION}

Cette étude nous a permis de mettre en évidence six ensembles magnétostratigraphiques (EMS) de susceptibilité différente, caractérisés par des propriétés magnétiques distinctes (tabl. 4). Chacun de ces ensembles magnétostratigraphiques incluant des niveaux archéologiques, il est important de tenir compte de la présence de l'Homme dans ce site préhistorique, celui-ci ayant pu modifier les caractéristiques lithologiques, pétrographiques et géochimiques des sédiments.

Dans ce qui suit, et pour chaque ensemble magnétostratigraphique (en partant de la base), après une brève description de leurs caractéristiques sédimentologiques, nous allons détailler chacun des paramètres évoqués plus haut, et, dans la mesure du possible, en proposer une interprétation. Des références à l'étude palynologique de Fellag (1996, 1998) sont fréquemment effectuées. Celles-ci, même si elles peuvent être discutées, permettent bien souvent de corroborer nos propres interprétations.

Le tableau 4 reprend les valeurs des principaux paramètres magnétiques pour chaque ensemble.

Exposé des résultats :

\section{Ensemble magnétostratigraphique (EMS) 1 :}

Epais de $72 \mathrm{~cm}$ (-855 à $-783 \mathrm{~cm})$, il est inclus dans l'unité stratigraphique $f$ de Farrand (éboulis de base) et correspond à la partie inférieure du remplissage (fig. 3). II ne contient aucun niveau archéologique. II s'est formé sur place par l'accumulation des cailloux et blocs tombés de la paroi calcaire sous un climat froid. Ces résultats sont corroborés par ceux de l'étude palynologique effectuée par Fellag en 1996, dans laquelle il montre que les pollens sont représentés presque exclusivement par des herbacées xérophiles et/ou steppiques qui témoignent de conditions rigoureuses (climat froid).

II s'agit d'un niveau sableux riche en cailloux, de couleur brun clair, qui présente un fort pourcentage en $\mathrm{CaCO}_{3}$ (matière diamagnétique qui donne de très faibles valeurs en susceptibilité magnétique) (Djerrab 2001).

Les valeurs de $\chi_{b f}$ et $\kappa_{b f}$ (fig. 4) sont très faibles, de même que celles de $\chi_{\mathrm{fd}}$. II est important de savoir que les résultats de ce dernier paramètre ne sont pas fiables, car il s'agit d'échantillons à très faible signal magnétique, et de plus, la sonde utilisée, de type Bartington, n'est pas assez sensible. La variation de $\chi_{\mathrm{fd}}$ n'apporte pas de renseignements sur la nature des grains magnétiques (Forster et al. 1994). Cependant, des valeurs de $\chi_{\mathrm{fd}}$ supérieures à $10 \%$, inexistantes dans notre étude, attesteraient de la dominance de grains SP (Thompson et Oldfield 1986).

Les deux paramètres magnétiques $\mathrm{ARI}_{\mathrm{s}}$ et $\mathrm{ARA}$ (fig. 4) présentent de très faibles valeurs, qui sont probablement dues à une faible concentration en oxydes de fer. Les valeurs du rapport $A R I-100 / A R I_{s}$ sont très faibles (inférieures à 0,5) [fig. 5]. Ce résultat confirme la dominance des grains magnétiques d'origine détritique (Lanci et al. 1999).

\begin{tabular}{|c|c|c|c|c|c|c|c|c|c|}
\hline $\begin{array}{c}\text { Ensembles } \\
\text { magnéto- } \\
\text { stratigraphiques }\end{array}$ & $\begin{array}{l}\text { Profondeur } \\
\text { en cm }\end{array}$ & $\begin{array}{l}\chi_{n+1} 10^{c}- \\
8 m^{3} / \mathbf{k g}\end{array}$ & $\gamma_{111} \%$ & $\begin{array}{l}\mathrm{ARI_{s }} \\
\mathrm{mA} / \mathrm{m}\end{array}$ & $\begin{array}{c}\mathrm{ART}- \\
100 \\
\mathrm{~mL} / \mathrm{m}\end{array}$ & $\begin{array}{c}\mathrm{ARA} \\
\mathrm{mA} / \mathrm{m}\end{array}$ & $\begin{array}{c}\text { ARl } / / \gamma_{\text {ht }} \\
\text { KA/mi }\end{array}$ & $\mathrm{ARI}_{3} / \mathrm{ARA}$ & $\mathrm{K}_{\mathrm{xrm} / \mathrm{K}} / \mathrm{K}_{\mathrm{nt}}$ \\
\hline 1 & -855 u -783 & 1,18 & 0 & 185 & 46,7 & 8 & 15,03 & 31.28 & 0,00484 \\
\hline 2 & $-78.3+63.30$ & 16,41 & $3-7$ & 1800 & 1800 & 150 & 11,82 & 12 & 0,00619 \\
\hline 3 & $-630 \div-500$ & 2,65 & $0-4$ & 500 & 208 & 22 & 17,73 & 91 & 0,00581 \\
\hline 4 & -500 id -285 & 38,60 & $5-9$ & 5887 & 5022 & 664 & 12,73 & 14.45 & 0,00535 \\
\hline 5 & -285 d -240 & 3,34 & 0 & $5 \%)$ & 333 & 40 & 14,71 & 20) & 0,00479 \\
\hline 6 & $-240 a-75$ & 23,54 & $0-7$ & 3600 & 3235 & 323 & 15,20 & 12.52 & 0,00814 \\
\hline
\end{tabular}
Le rapport $A R I_{s} / \chi_{\text {bf }}$ (fig. 5) présente de fortes valeurs, ce qui

Tableau 4 - Résultats des différents paramètres magnétiques.

Les valeurs de $\chi_{b f}, A R I_{s}, A R I-100, A R A, A R I_{s} / \chi_{b f}, A R I_{s} / A R A$ et de $K_{a m} / K_{b f}$ sont des valeurs moyennes.

Table 4 - Results of magnetic parameters. 


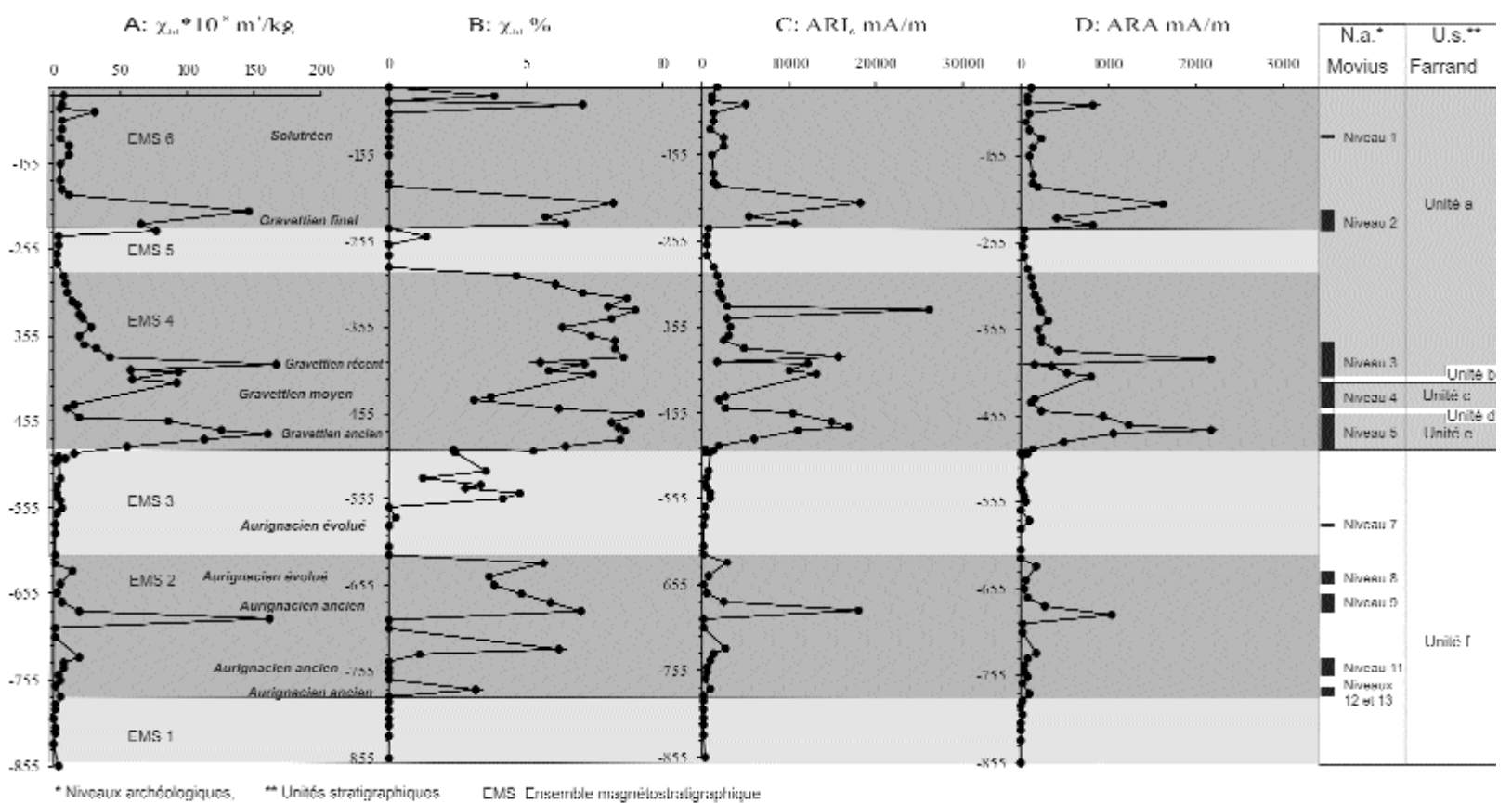

Figure 4 - Mesure des paramètres magnétiques des échantillons prélevés à l'Abri Pataud : A : Susceptibilité magnétique massique à basse fréquence (10-8 m3/kg) ; B : Dépendance en fréquence de la susceptibilité magnétique (\%) ; $C$ : Aimantation Rémanente Isotherme de Saturation $(\mathrm{mA} / \mathrm{m}) ; D$ : Aimantation Rémanente Anhystérétique $(\mathrm{mA} / \mathrm{m})$.

Figure 4 - Magnetic parameters of the Abri Pataud samples: A: Specific magnetic susceptibility at low frequency (10-8 m3/kg); $B$ : Frequency dependence of magnetic susceptibility (\%); C: Isothermal Remanent Magnetization to Saturation (mA/m); $D$ : Anhysteretic Remanent Magnetisation $(\mathrm{mA} / \mathrm{m})$.

pourait indiquer la faible concentration en éléments magnétiques de taille fine (magnétite et maghémite). Les valeurs de ce rapport diminuent quand la taille des grains augmente (uniquement pour les grains dont la taille est supérieure à la taille limite SP / MD) (Stockhausen et al., 1999). Les grains SP ne portent pas de rémanence (rémanence nulle), et donc ne contribuent pas aux valeurs de $\mathrm{I}^{\prime} \mathrm{ARI} \mathrm{s}_{\mathrm{s}}$. Par contre, leur présence dans les sédiments provoque une augmentation des valeurs de la susceptibilité magnétique.

Les fortes valeurs du rapport $A R I_{s} / \chi_{b f}$ mettent en évidence l'abondance des grains magnétiques d'origine détritique. Le rapport $A R I_{s} / A R A$ (fig. 5) présente de fortes valeurs. II faut savoir que de faibles valeurs de ce rapport indiquent que la taille des grains magnétiques est très fine (Banerjee et al. 1981 ; Maher 1988), tandis que dans le cas de la dominance de grains $M D$, les valeurs de ce rapport se rapprochent de 1. Dans notre cas, les valeurs sont fortes, ce qui pourrait indiquer la dominance de grains PD. Les grains de petite taille donnent de hautes valeurs de l'ARA parce qu'ils sont très efficaces pendant l'acquisition de la rémanence, particulièrement les grains MD (Maher 1988 ; Dunlop 1995).

Les valeurs de $\mathrm{K}_{\mathrm{am}}$ sont faibles (grains magnétiques peu abondants), de même que le rapport $\mathrm{K}_{\mathrm{am}} / \mathrm{K}_{\mathrm{bf}}$ (fig. 6). $\mathrm{K}_{\mathrm{arm}}$ et $\kappa_{b f}$ évoluent de façon continue, mais en sens inverse : $\kappa_{b f}$ augmente, tandis que $\mathrm{K}_{\mathrm{am}}$ diminue quand la taille de grains magnétiques augmente (Collombat 1993). Le rapport $\mathrm{K}_{\mathrm{arm}} / \kappa_{\mathrm{bf}}$ reflète donc les variations relatives de taille des grains (Banerjee et al. 1981 ; King et al. 1982 ; Hartstra 1982 ; Bloemendal et al. 1992). Ainsi, les faibles valeurs obtenues pour ce rapport indiquent la dominance des grains PD.

Pour résumer, les grains magnétiques présents dans cet ensemble magnétostratigraphique d'éboulis sont d'origine détritique (c'est-à-dire résultant de la désagrégation mécanique et ne s'exposant à aucune forme d'évolution géochimique postérieure) et sont dominés par des grains de fort champ coercitif, tels que l'hématite, et probablement la goethite. La magnétite, de taille $P D$, n'est présente qu'en faible quantité. Sous un climat froid et sec, les carbonates de fer présents dans les roches calcaires (sidérite) et les oxydes de fer restent à l'état primaire et ne se transforment pas en oxydes de fer secondaires (pédogenèse nulle).

Ensemble magnétostratigraphique (EMS) 2 : Epais de $153 \mathrm{~cm}(-783$ à $-630 \mathrm{~cm})$ [fig.4], il se présente comme un niveau sablo-limoneux riche en cailloux, de couleur brun foncé à brun clair. Deux lits limoneux de couleur brun foncé sont visibles.

Les valeurs de $\chi_{b f}$ sont plus élevées que dans le niveau sous-jacent ( 1 à $162^{*} 10^{-8} \mathrm{~m}^{3} / \mathrm{kg}$ ) (fig. 4), et la présence de quelques pics de susceptibilité, par exemple à $-685 \mathrm{~cm}$ $\left(162^{*} 10^{-8} \mathrm{~m}^{3} / \mathrm{kg}\right)$, est liée à celle des niveaux contenant de nombreux éléments chauffés. En effet, les foyers et leurs zones de vidange, reconnus sur le terrain par la présence de charbon et de couleurs sombres, donnent de fortes valeurs de $\kappa_{\mathrm{bf}}$ (mesurée directement sur les coupes). 


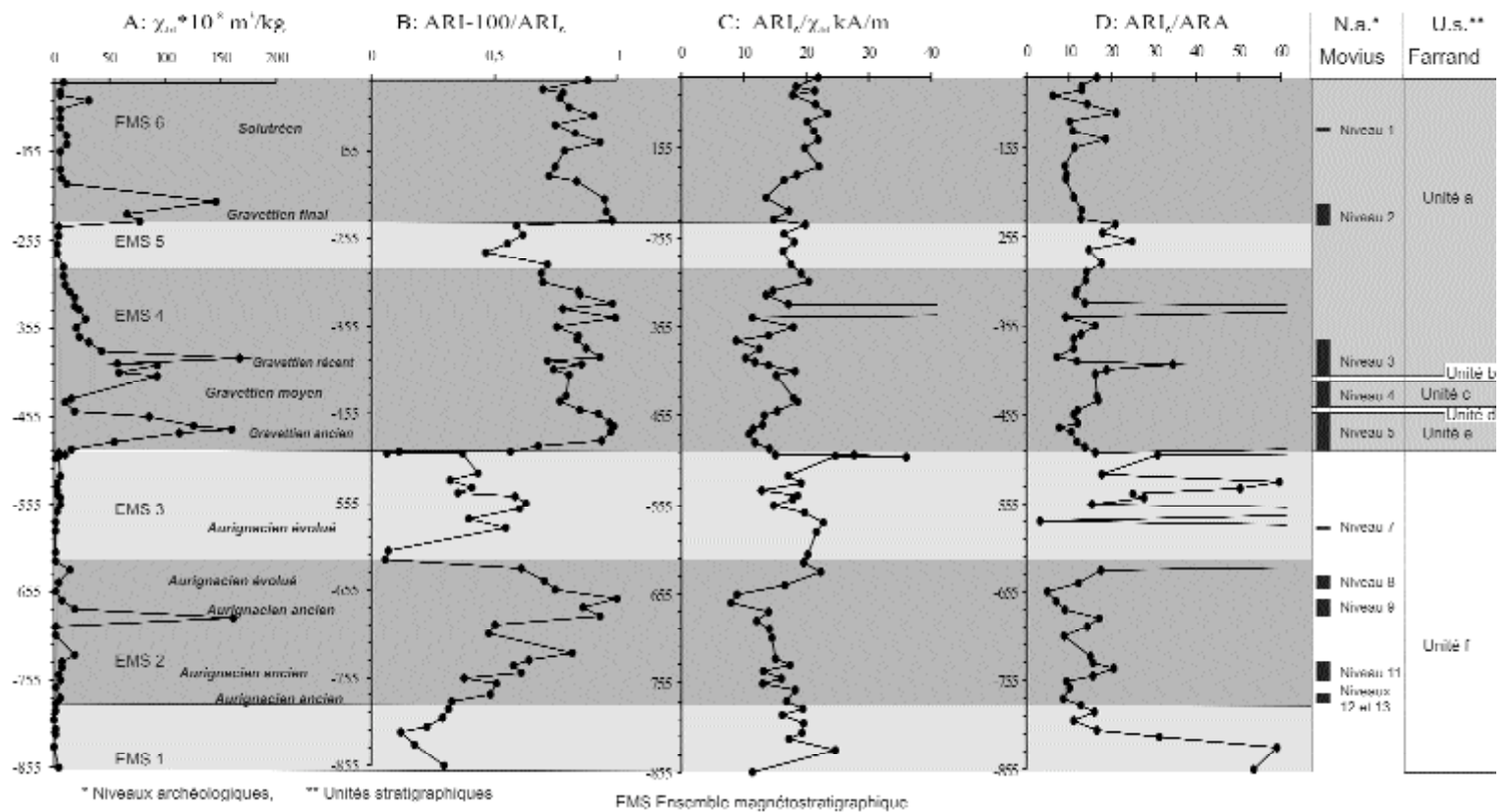

Figure 5 - Mesure des paramètres magnétiques des échantillons prélevés à l'Abri Pataud : A : Susceptibilité magnétique massique à basse fréquence (10-8 m3/kg); B : Rapport de l'Aimantation Rémanente lsotherme à -100 mT et de l'Aimantation Rémanente Isotherme de saturation à $1000 \mathrm{mT} ; \mathrm{C}:$ Rapport ARIs/cbf $(\mathrm{kA} / \mathrm{m}) ; D$ : Rapport ARIs/ARA.

Figure 5 - Magnetic parameters of the Abri Pataud samples: A: Specific magnetic susceptibility at low frequency (10-8 $\mathrm{m} 3 / \mathrm{kg})$; B: ARI-100/ARIs ratio; C: ARIs /_bf ratio kA/m; D: ARIs/ARA ratio.

Cet ensemble magnétostratiographique, inclus dans l'unité stratigraphique $f$ de Farrand, contient 6 niveaux archéologiques aurignaciens, de couleur noire ou ocrée. Le niveau archéologique 13 (Aurignacien ancien), daté de $34000 \pm 675$ BP (laboratoire de Groningen in Movius, 1975) correspond à la légère augmentation de $\chi_{\text {bf }}$ enregistrée à $-775 \mathrm{~cm}\left(5^{\star} 10^{-8}\right.$ $\mathrm{m}^{3} / \mathrm{kg}$ ).

Le niveau archéologique 12 (Aurignacien ancien), daté de $33260 \pm 500$ BP (laboratoire de Groningen), se localise entre -760 et $-766 \mathrm{~cm}$.

Le niveau archéologique 11 (Aurignacien ancien), daté de $32600 \pm 800$ BP (laboratoire de Groningen), se localise entre -750 et $-730 \mathrm{~cm}$. $\chi_{\mathrm{fd}}$ est très faible (0 à $7 \%$ ) (fig. 4). Les niveaux archéologiques 10 et 9 (Aurignacien ancien), ainsi que le niveau 8 (Aurignacien évolué), ne sont pas décelables.

$\chi_{\mathrm{fd}}$ est très faible dans les niveaux clairs, qu'ils soient stériles ou riches en matériel archéologique, et plus forte (7\%) dans les niveaux archéologiques (foncés), riches en éléments brûlés. Ce résultat révèle l'absence de grains SP dans les niveaux clairs, et la présence d'un mélange de grains de taille variée dans les autres niveaux, notamment au sein des foyers (nous appliquons le terme foyer aux sédiments brulés in situ ou redistribués lors de la vidange des foyers)

Les valeurs de l'ARI $\mathrm{s}_{\mathrm{s}}$, comprises entre 200 et $17000 \mathrm{~mA} / \mathrm{m}$, sont relativement fortes (fig. 4). La courbe de l'ARA présente une allure similaire à celle de l'ARI ${ }_{s}$, avec des valeurs comprises entre 17 et $280 \mathrm{~mA} / \mathrm{m}$ (fig. 4).
Le rapport $A R I-100 / A R I_{s}(0,4$ à 1$)$ est très faible dans les niveaux clairs et plus fort dans les niveaux archéologiques (fig. 5). Les grains magnétiques présents dans les premiers niveaux saturent difficilement et ont un fort champ coerc itif. Au contraire, les grains magnétiques des niveaux sombres saturent plus rapidement et plus facilement.

Le rapport $A R I_{s} / \chi_{b f}$, est élevé dans les niveaux clairs, et plus faible dans les niveaux archéologiques riches en matériels brulés, ce qui signifie que la taille des grains magnétiques diminue au niveau des couches archéologiques.

Au contraire, les valeurs de $\mathrm{K}_{\mathrm{am}}$ et du rapport $\mathrm{K}_{\mathrm{am}} / \mathrm{K}_{\mathrm{bf}}$ sont fortes dans les niveaux archéologiques riches en matériels brulés et faibles dans les niveaux clairs (fig. 6).

Les grains magnétiques présents dans les niveaux sablolimoneux de couleur brun clair sont identiques à ceux contenus dans l'ensemble magnétostratigraphique 1, c'est-à-dire qu'il s'agit de grains de fort champ coercitif, à faible signal magnétique, d'origine détritique. La présence de ces seuls grains détritiques indique que la fraction magnétique est héritée de l'encaissant à la suite de la désagrégation de ce demier sous climat froid. Cette interprétation paléoenvironnementale est confirmée par l'étude palynologique qui montre la dominance de taxons steppiques de climat froid, donc un climat froid (Armaranthacées-Chénopodiacées, Astéracées type fenestré et échinulé).

Les principaux oxydes de fer sont l'hématite, accompagnée probablement de goethite et de grains $P D$ de magnétite.

$A u$ contraire, les grains magnétiques du niveau archéologique 12 sont d'origine secondaire, issus de la transforma- 


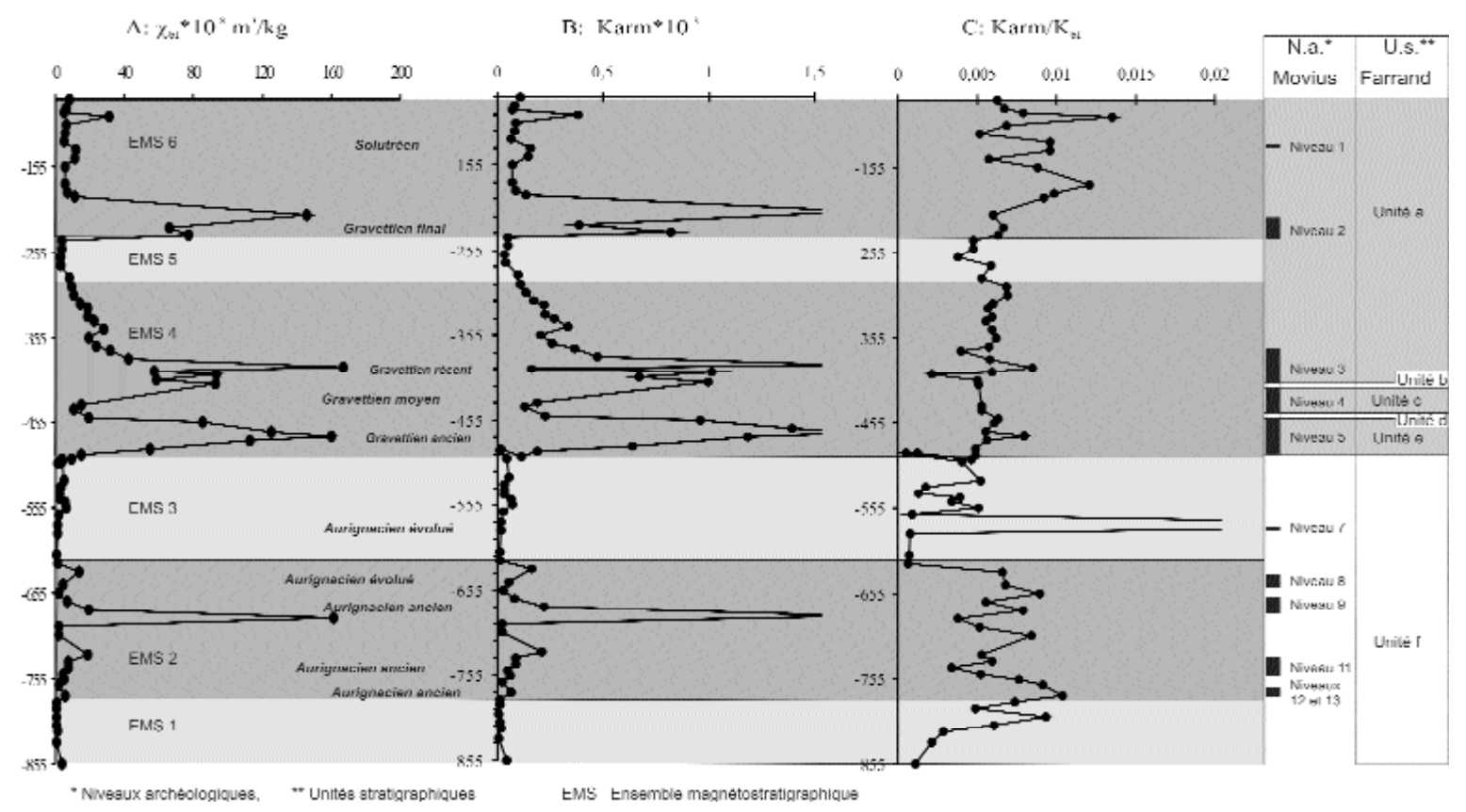

Figure 6 - Mesure des paramètres magnétiques des échantillons prélevés à l'abri Pataud : $A$ : Susceptibilité magnétique massique à basse fréquence (10-8 m3/kg) ; B : Susceptibilité Anhystérétique (10-3 S.I) ; C : Rapport de la susceptibilité anhystérétique et de la susceptibilité magnétique.

Figure 6 - Magnetic parameters of the Abri Pataud samples: A: Specific magnetic susceptibility at low frequency (10-8 m3/kg); B: Anhysteretic magnetic susceptibility (10-3 S.I); C : Karm/Kbf ratio.

tion des oxydes de fer primaires par des phénomènes complexes d'oxydation - réduction (transformation des oxydes de fer suite aux températures élevées dans les foyers). Ces grains magnétiques ont un faible champ coercitif, il s'agit essentiellement de magnétite, de maghémite et probablement d'hématite. La taille de ces grains est variable, ils semblent cependant dominés par les grains SP et PMD. La présence de sédiments contenant de nombreux éléments chauffés est attestée par leur couleur, la présence visible de cendre et de charbon et bien entendu par les fortes valeurs des différents paramètres magnétiques.

Ensemble magnétostratigraphique (EMS) 3 : D'une épaisseur de $130 \mathrm{~cm}(-630$ à $-500 \mathrm{~cm}$ ) (fig. 4), il s'agit d'un ensemble d'éboulis de couleur claire, riche en sable, qui contient un niveau archéologique aurignacien évolué : le niveau 7. Ce dernier n'est pas décelable en termes de susceptibilité magnétique. Les valeurs des différents paramètres magnétiques mesurées sont très faibles et identiques à celles de l'ensemble magnétostratigraphique 1 .

Le diagramme de Lowrie de l'échantillon AP-65 (niveau archéologique 7) (fig. 7), montre l'évolution de l'ARI en fonction de la température selon les trois axes $X$ (champ coercitif faible), $Y$ (champ coercitif moyen) et $Z$ (champ coercitif fort). L'ARI selon l'axe $X$ montre une rupture de pente à $200^{\circ} \mathrm{C}$, qui révèle probablement la présence de goethite $(\alpha \mathrm{FeOOH})$ ou de titanomagnétite. La goethite est un hydroxyde de fer, antiferromagnétique (accompagné d'un faible ferromagnétisme fortement anisotrope
(Rochette et Fillion 1989). Elle est instable à partir de 180$400{ }^{\circ} \mathrm{C}$ et se déshydrate en hématite (Hedley 1968 ; Özdemir et Dunlop 1996 ; Ruan et al. 2001 ; Przepiera et Przepiera 2003). Une deuxième chute, moins importante, est observée entre 300 et $400{ }^{\circ} \mathrm{C}$ (transformation de pyrrhotite ou de maghémite). La courbe de l'ARI selon l'axe $X$ poursuit sa chute et s'annule à $580{ }^{\circ} \mathrm{C}$ (point de Curie de la magnétite). Tout comme l'ARI selon l'axe $X$, I'ARI selon l'axe $\mathrm{Y}$ diminue entre 300 et $400{ }^{\circ} \mathrm{C}$ (transformation soit de pyrite, de pyrrhotite ou de maghémite). Quant à l'ARI selon l'axe $Z$, après une chute brutale à $100^{\circ} \mathrm{C}$ (transformation de goethite, présente dans certains calcaires (Heller 1978 ; Lowrie et Heller 1982), elle chute une deuxième fois entre 300 et $400^{\circ} \mathrm{C}$.

La courbe d'acquisition de l'aimantation à saturation de cet échantillon (fig. 8) montre que les grains magnétiques ne saturent pas à $100 \%$, même en présence d'un champ magnétique fort (1 T), ce qui reflète la présence des composantes magnétiques de fort champ coercitif (hématite et goethite).

Le diagramme proposé par Day et al. en 1977, modifié (Dunlop, 2002), (fig. 9) $\left(\mathrm{M}_{\mathrm{rs}} / \mathrm{M}_{\mathrm{s}}\right.$ en fonction de $\left.\mathrm{H}_{\mathrm{cr}} / \mathrm{H}_{\mathrm{c}}\right)$ montre que l'échantillon AP-63 (éboulis 5/7), se localise dans la zone caractéristique des grains PD. La susceptibilité ferromagnétique $\left(\kappa_{\mathrm{feno}}\right)$, mesurée à partir du cycle d'hystérésis, représente plus de $85 \%$ de la valeur totale de $\kappa_{0}$. La valeur des susceptibilités $\kappa_{\text {par }}+\kappa_{\text {dia }}+\kappa_{\text {antiferro }}$ est relativement importante (plus de $15 \%$ ). 

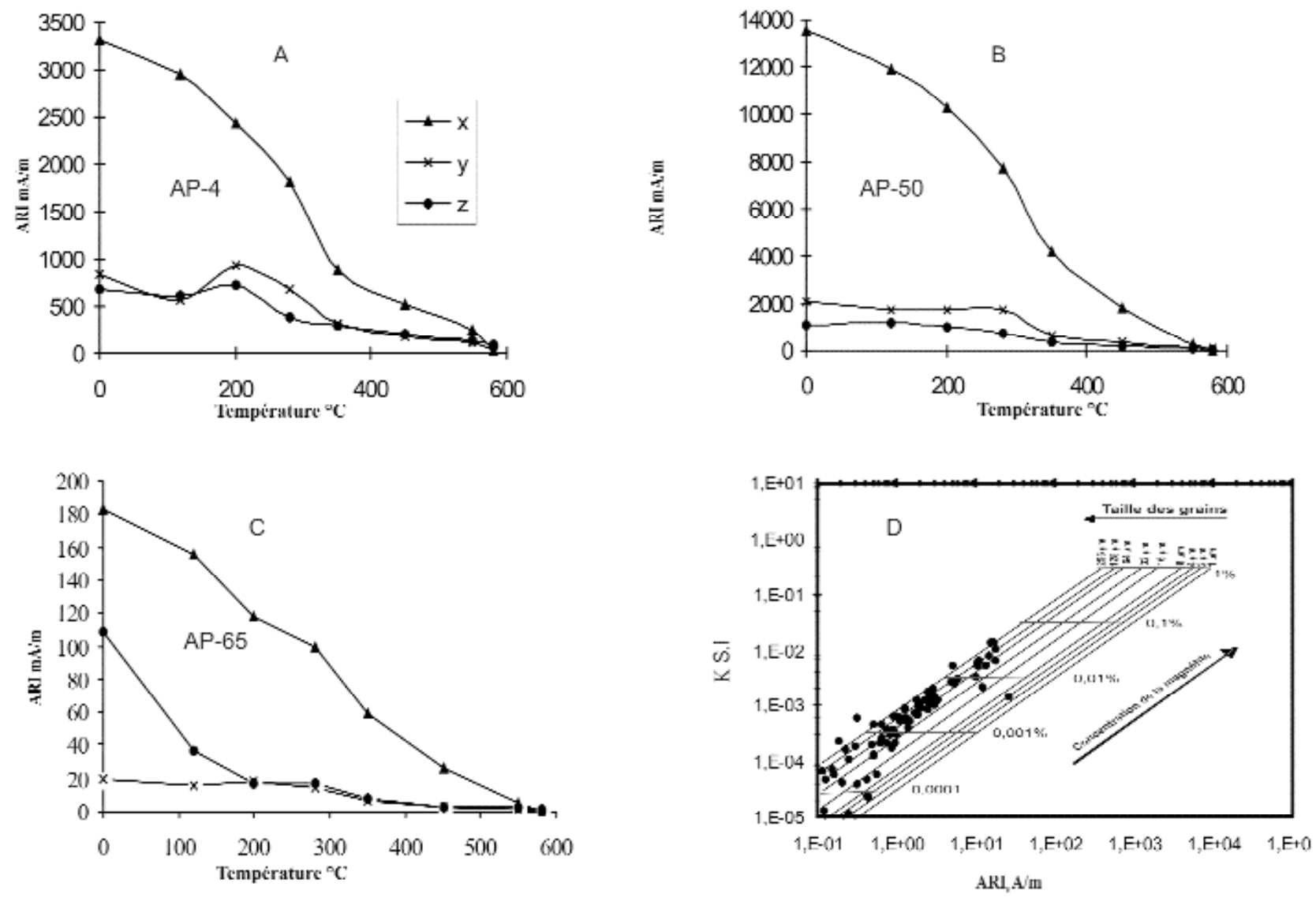

Figure 7 - Diagrammes de Lowrie des échantillons AP-4, AP-50, AP-65 et diagramme bi-logarithmique de la susceptibilité magnétique en fonction de l'Aimantation Rémanente Isotherme de saturation.

Figure 7 - Lowrie diagrams of the samples AP-4, AP-50, AP-65, and Bilogarithmic diagram of Isothermal Remanence Magnetization to saturation versus magnetic susceptibility.

En conclusion, cet ensemble magnétostratigraphique présente des propriétés magnétiques semblables à celles de l'ensemble magnétostratigraphique 1. Les grains magnétiques présents sont d'origine primaire, essentiellement composés d'hématite et de goethite. Les valeurs de la susceptibilité magnétique sont contrôlées par les minéraux antiferromagnétiques et diamagnétiques.

Ensemble magnétostratigraphique (EMS) 4 : Epais de $215 \mathrm{~cm}(-500 \mathrm{à}-285 \mathrm{~cm})$ [fig. 4], il peut être décomposé en plusieurs zones :

- sables jaunes micacés dans la partie inférieure (- 500 à $490 \mathrm{~cm}$ ) [coupe stratigraphique transversale 69/70]. Cette zone se situe dans l'éboulis $5 / 7$ de Movius ;

- sédiments bruns rouges à bruns foncés, cendreux, riches en matériel archéologique $(-490$ à $-450 \mathrm{~cm})$. Cette zone inclut le niveau archéologique 5 attribué au Gravettien ancien :

- sédiment limoneux de couleur jaune clair, très caillouteux, surtout à la base, où on note également la présence de radicelles (450 à - $435 \mathrm{~cm}$ ) [partie supérieure de la coupe stratigraphique transversale 69/70]. Cette zone inclut le niveau archéologique 4 attribué au Gravettien moyen ; - sédiment sablo-limoneuse de couleur brun foncée, à rares cailloux, riche en en matériel archéologique et en charbon (435 à $-285 \mathrm{~cm}$ ) [base de la coupe 70/71]. Cette zone inclut le niveau archéologique 4 attribué au Gravettien moyen et le niveau 3 attribué au Gravettien récent ;

Les valeurs de $\chi_{\mathrm{bf}}$ sont élevées (54 à $160^{*} 10^{-8} \mathrm{~m}^{3} / \mathrm{kg}$ (fig. 4), ce qui révèle la présence d'une concentration importante en oxydes de fer. $\chi_{\mathrm{fd}}$ confime la présence d'un mélange de grains magnétiques de taille variable, toutefois dominés par des grains fins, plus particulièrement à la base du niveau (fig. 4). Les valeurs de l'ARI $(6000$ à $17000 \mathrm{~mA} / \mathrm{m}$ ) et de l'ARA (500 à $2200 \mathrm{~mA} / \mathrm{m}$ ) sont fortes, ce qui suggère une importante concentration en grains magnétiques. Les grains magnétiques saturent à $90 \%$ en présence d'un champ magnétique faible $(100 \mathrm{mT})$.

Les faibles valeurs des deux rapports $A R I_{S} / \chi_{b f}$ et $A R I_{S}$ /ARA indiquent la dominance de grains magnétiques 

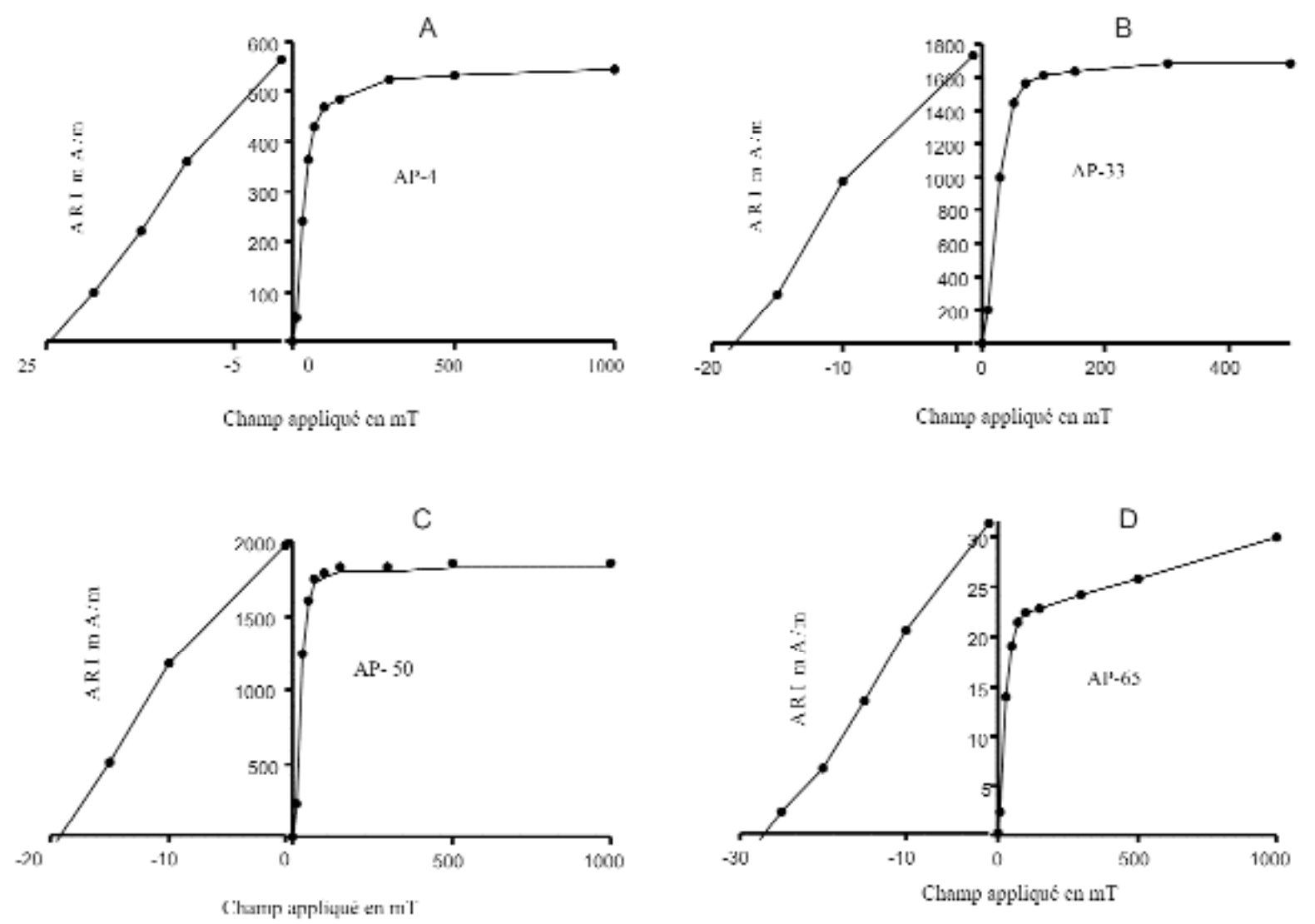

Figure 8 - Courbes d'acquisition de l'aimantation rémanente à saturation des échantillons $A P-4, A P-33, A P-50$ et $A P-65$ en fonction du champ appliqué.

Figure 8 - Acquisition curves of IRM to saturation of the samples AP-4, AP-33, AP-50 and AP-65 versus applied field.

de taille fine (SP, PMD et MD), attestation qui est renforcée par les fortes valeurs de $\mathrm{K}_{\mathrm{am}}$ et du rapport $\mathrm{K}_{\mathrm{am}} / \mathrm{K}_{\mathrm{bf}}$.

Le diagramme de Lowrie de l'échantillon AP-50, (niveau archéologique 5) (fig. 7), confirme la dominance de maghémite, selon les trois axes $(X, Y$ et $Z)$. Selon l'axe $X$, on signale une diminution importante de l'ARI entre 300 et $400{ }^{\circ} \mathrm{C}$ (transformation de la maghémite ou de la pyrrhotite), puis la courbe continue à diminuer jusqu'à $580^{\circ} \mathrm{C}$ (point de Curie de la magnétite). On n'observe aucune trace ni d'hématite, ni de goethite. L'ARI selon l'axe Y montre également la présence de maghémite, la courbe est en effet marquée par la même forte rupture de pente entre 300 et $400^{\circ} \mathrm{C}$. L'ARI selon l'axe $Z$ révèle aussi la dominance de maghémite. La courbe d'acquisition de l'aimantation à saturation du même échantillon (fig. 8) présente une saturation de plus de $90 \%$ des grains magnétiques dans un champ magnétique inférieur à $100 \mathrm{mT}$. La saturation totale des grains magnétiques dans un champ magnétique inférieur à 200 mT est une caractéristique de la dominance de maghémite ou de magnétite. Le champ coercitif de saturation $\left(B_{0}\right)_{c r}$ est faible (inférieur à 20 mT).
Quant à la courbe d'acquisition de l'aimantation à saturation de l'échantillon AP-33 (niveau archéologique 3) (fig. 8), elle montre des propriétés magnétiques identiques à celles de l'échantillon AP-50.

Le diagramme proposé par Day et al. en $1977\left(\mathrm{M}_{\mathrm{rs}} / \mathrm{M}_{\mathrm{s}}\right.$ en fonction de $\mathrm{H}_{\mathrm{cr}} / \mathrm{H}_{\mathrm{c}}$ ) montre que l'échantillon AP-49 (niveau archéologique 5), se localise dans la zone dominée par les grains PMD (fig. 9). Cet échantillon, prélevé dans les sédiments contenant de nombreux éléments chauffés, est caractérisé par un faible champ coercitif de rémanence et par de fortes valeurs de $M_{r s}$, ce qui semble être dû à une forte concentration en grains magnétiques (magnétite et maghémite). Son cycle d'hystérésis montre la nette dominance de la susceptibilité ferromagnétique (plus de $99 \%$ ). II est à signaler que la formation de maghémite dans les foyers a été démontrée par Le Borgne (1955, 1964, 1965) et Grogan et al. (2003).

L'échantillon AP-34 (niveau archéologique 3), se localise dans une zone dominée par les grains PD $80 \%$ suivi par les grains MD et PMD 20\% (fig. 9). Le cycle d'hystérésis 


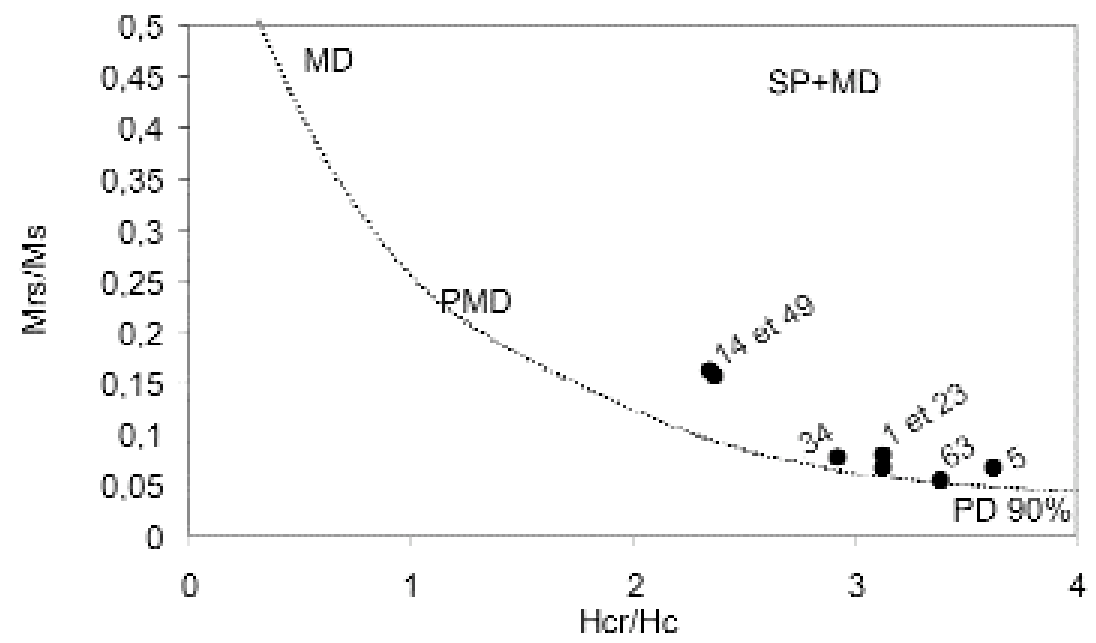

Figure 9 - Diagramme du rapport $\mathrm{Mr} / \mathrm{Ms}$ en fonction de $\mathrm{Hcr} / \mathrm{Hc}$ (d'après Day et al., 1977, modifié par Dunlop 2002).

Figure 9 - Day plot of Mrs/Ms versus Hcr/Hc (Day et al., 1977, modified by Dunlop 2002).

montre la dominance de la susceptibilité ferromagnétique (plus de $84 \%$ ), avec malgré tout une contribution importante des susceptibilités diamagnétique, paramagnétique et antiferromagnétique (plus de $15 \%$ ).

Enfin l'échantillon AP-23 (éboulis 2/3), se localise dans la zone dominée par les grains PD $85 \%$. Le champ coercitif de rémanence est de l'ordre de 29,30 mT (23,85 kA/m). Le pourcentage de grains magnétiques est plus faible que pour l'échantillon AP-14. La susceptibilité ferromagnétique présente plus de $91 \%$ de la valeur totale de $\kappa_{0}$.

Cet ensemble magnétostratigraphique est caractérisé par la présence de foyers et de plusieurs niveaux archéologiques (Movius 1975 ; Nespoulet et Chiotti 2007b). Les fortes valeurs des différents paramètres magnétiques confirment l'abondance de grains magnétiques de taille variable, dominés toutefois par ceux de taille MD et SP. Sous l'effet de la température élevée des foyers, les oxydes de fer primaires et les carbonates de fer sont transformés en oxydes de fer secondaires. Par conséquent, le signal magnétique est contrôlé par la magnétite et la maghémite. En dehors des foyers et des zones de vidange de ces derniers, cet ensemble magnétostratigraphique, constitué d'éboulis, est identique aux ensembles magnétostratigraphiques 3 et 1.

Ensemble magnétostratigraphique (EMS) 5 : Ensemble dominé par les éboulis, épais de $45 \mathrm{~cm}(-285$ à $-240 \mathrm{~cm})$ (partie supérieure de la coupe stratigraphique transversale 72/73). II se situe dans l'éboulis 2/3 de Movius.

L'ensemble des paramètres magnétiques présente de faibles valeurs et révèle une faible concentration en grains magnétiques.

$\chi_{\text {fd }}$ (inférieur à $4 \%$ ) est plus faible que dans le niveau sousjacent, ce qui pourrait indiquer la présence d'un faible pourcentage en grains magnétiques de taille fine (fig. 4). Les courbes des deux paramètres magnétiques $\left(\mathrm{ARI}_{\mathrm{s}}\right.$ et ARA) évoluent d'une manière identique à celle de $\chi_{b f}$. En observant les valeurs du rapport ARI-100/ARI , on constate que les grains magnétiques ne saturent pas à $100 \%$, ce qui est une caractéristique de la présence de grains goethite ou d'hématite (Djerrab 2001).
Les valeurs des différents paramètres magnétiques de cet ensemble magnétostratigraphique sont légèrement supérieures à celles des ensembles magnétostratigraphiques 1 et 3 (tabl. 4), mais cette différence est peu significative. Ces trois ensembles magnétostratigraphiques se sont formés sous des conditions climatiques très proches, et l'augmentation observée ici peut s'expliquer par un pourcentage plus élevé de grains magnétiques d'origine détritique.

Ensemble magnétostratigraphique (EMS) 6 : II occupe la partie supérieure du remplissage. Plusieurs zones sont distinguées :

- des éboulis calcaires jaune pâle, formés de sédiments relativement fins, contenant quelques charbons de bois, quelques lits de terre noirâtre et de terre rubéfiée (sédiments contenant de nombreux éléments chauffés). Cette zone occupe la partie supérieure de la coupe stratigraphique transversale $74 / 75$ et la partie inférieure de la coupe stratigraphique transversale 76/77 (-240 à - $125 \mathrm{~cm})$. Elle comespond à l'éboulis $1 / 2$ de Movius et au niveau archéologique 2 (Gravettien final) ;

- un petit lit de terre rubéfiée $(-125$ à - $120 \mathrm{~cm})$, contenant des traces de charbon et des radicelles. Cette zone correspond au niveau archéologique 1 (Solutréen), daté de $20400 \pm 450$ BP ;

- des éboulis calcaires jaune pâle $(-120 \mathrm{~cm}$ à - $75 \mathrm{~cm})$ (partie supérieure de la coupe stratigraphique transversale 76/77). Cette zone correspond aux éboulis stériles situés au-dessus du niveau archéologique 1.

$\chi_{b f}$, comprise entre 5 et $145.68^{*} 10^{-8} \mathrm{~m}^{3} / \mathrm{kg}$ (fig. 4), est plus élevée que dans le niveau sous-jacent et augmente légèrement à $-135 \mathrm{~cm}$. Les deux paramètres magnétiques $A R I_{s}$ et ARA présentent de faibles valeurs dans les niveaux à éboulis et de fortes valeurs dans les foyers et les paléosols, et confirment les résultats de $\chi_{\mathrm{bf}}$. Les grains magnétiques saturent plus rapidement que ceux du niveau sousjacent, mais, en présence d'un champ moyennement fort $(500 \mathrm{mT})$, tous les grains magnétiques saturent à $100 \%$. Le rapport $A R I-100 / A R I_{s}$ se rapproche de 1 . Les oxydes de fer ont un champ coercitif moyen. 
Les deux rapports $A R I_{s} / \chi_{b f}$ et $A R I_{s} / A R A$ révèlent la présence d'un mélange de grains SP, PMD, MD et PD. Les valeurs de $\mathrm{K}_{\mathrm{a} \text { rm }}$ et du rapport $\mathrm{K}_{\mathrm{am}} / \mathrm{K}_{\mathrm{bf}}$ augmentent légèrement et plus particulièrement à $-135 \mathrm{~cm}$ (fig. 6). II paraît évident que les grains magnétiques de cet ensemble magnétostratigraphique sont de taille variable, à l'exception de la zone comprise à la profondeur de -240 et -190 $\mathrm{cm}$, où les grains de taille fine dominent.

Le diagramme de Lowrie de l'échantillon AP-4 (éboulis 0/1) (fig. 7), montre une chute de l'ARI selon l'axe $X$ entre 300 et $400^{\circ} \mathrm{C}$. Cette chute pourrait indiquer la présence de pyrrhotite, et qui se transforme en magnétite. L'ARI poursuit sa chute et s'annule à $580^{\circ} \mathrm{C}$ (point de Curie de la magnétite). L'ARI selon les deux axes $Y$ et $Z$ indique probablement la présence de goethite (diminution de l'ARI entre 100 et $150^{\circ} \mathrm{C}$ ). Ensuite, l'ARI augmente à $200^{\circ} \mathrm{C}$, puis diminue à $300^{\circ} \mathrm{C}$ (transformation de lépidocrocite en maghémite, puis de maghémite en hématite), et enfin diminue légèrement à $580^{\circ} \mathrm{C}$ (point de Curie de la magnétite). Elle ne s'annule pas au-delà de $580^{\circ} \mathrm{C}$, ce qui prouve la présence d'un résidu d'hématite.

La courbe d'acquisition de l'aimantation à saturation du même échantillon (fig. 8) montre la dominance de la magnétite toutefois avec une faible présence d'hématite et de goethite. Le champ coercitif de saturation est moyen ( $25 \mathrm{mT})$.

Le diagramme proposé par Day et al. en $1977\left(\mathrm{M}_{\mathrm{rs}} / \mathrm{M}_{\mathrm{s}}\right.$ en fonction de $\mathrm{H}_{\mathrm{c}} / \mathrm{H}_{\mathrm{c}}$ ) montre que l'échantillon AP-14 (niveau archéologique 2) présente des propriétés magnétiques identiques à celles de l'échantillon AP-49 (fig. 9). Le rapport $H_{c r} / H_{c}$ présente une valeur proche de 2,37, ce qui révèle probablement la présence, soit de magnétite, soit de maghémite (Parry 1965 ; Rahman et al. 1973 ; Day et al. 1977 ; Parry 1981 ; Hartstra 1982 ; Özdemir et Banerjee 1982 ; Bailey et Dunlop 1983 ; Dunlop 1986). Le cycle d'hystérésis de cet échantillon montre une grande dominance de grains ferromagnétiques (SP et PMD). La susceptibilité ferromagnétique est très forte, elle représente plus de $99 \%$ de la susceptibilité totale.
Quant à l'échantillon AP-05 (éboulis 0/1) (fig. 9), il se localise dans une zone caractéristique des grains de taille $\mathrm{PD}$ (90\%). Le rapport $\mathrm{H}_{\mathrm{cr}} / \mathrm{H}_{\mathrm{c}}$ de cet échantillon est de l'ordre de 6,62. II contient probablement soit des grains PD de magnétite (Parry 1965 ; Rahman et al. 1973 ; Day et al. 1977 ; Parry 1981 ; Hartstra 1982 ; Özdemir et Banerjee 1982 ; Bailey et Dunlop 1983 ; Dunlop 1986), soit des grains de maghémite. Le champ coercitif de rémanence $\left(H_{c r}\right)$ de cet échantillon est de l'ordre de 31,28 mT (25,03 $k A / m)$, ce qui prouve la présence de grains PD de magnétite. La susceptibilité ferromagnétique $\left(\kappa_{\mathrm{feno}}\right)$, mesurée à partir du cycle d'hystérésis, représente plus de $92 \%$ de la susceptibilité totale de l'échantillon. Les résultats obtenus montrent donc la grande contribution des matières ferromagnétiques aux valeurs de $\kappa_{0}$.

Cet ensemble magnétostratigraphique, caractérisé par une légère hausse de la fraction fine (limon et argile), montre une concentration modérée en grains magnétiques d'origine secondaire (magnétite et maghémite). II s'agit principalement de grains magnétiques, de taille fine, formés sous l'effet de la pédogenèse (entre - 120 et - $125 \mathrm{~cm}$ ). En plus du phénomène de pédogenèse, certains oxydes de fer de ce niveau peuvent être issus de la combustion des sédiments et de matière organique dans les foyers.

Ci-après sont évoqués deux graphes, qui concernent des échantillons prélevés tout le long du remplissage (figures $7 D$ et 10). II s'agit des représentations de Thompson et Oldfield (1986) d'une part, et de King et al. (1982) d'autre part. Ces deux représentations permettent d'avoir une idée sur la taille et la concentration en grains magnétiques, la première étant utilisée en particulier pour la magnétite.

Sur le premier diagramme (variation de $\mathrm{k}$ en fonction de l'ARI $\mathrm{s}_{\mathrm{s}}$, fig.7D), on observe que les échantillons prélevés dans les niveaux sablo-limoneux riches en sédiments brûlés se localisent dans la zone de forte concentration en magnétite (entre 0.1 et $0.001 \%$ ). Au contraire, les échantillons prélevés

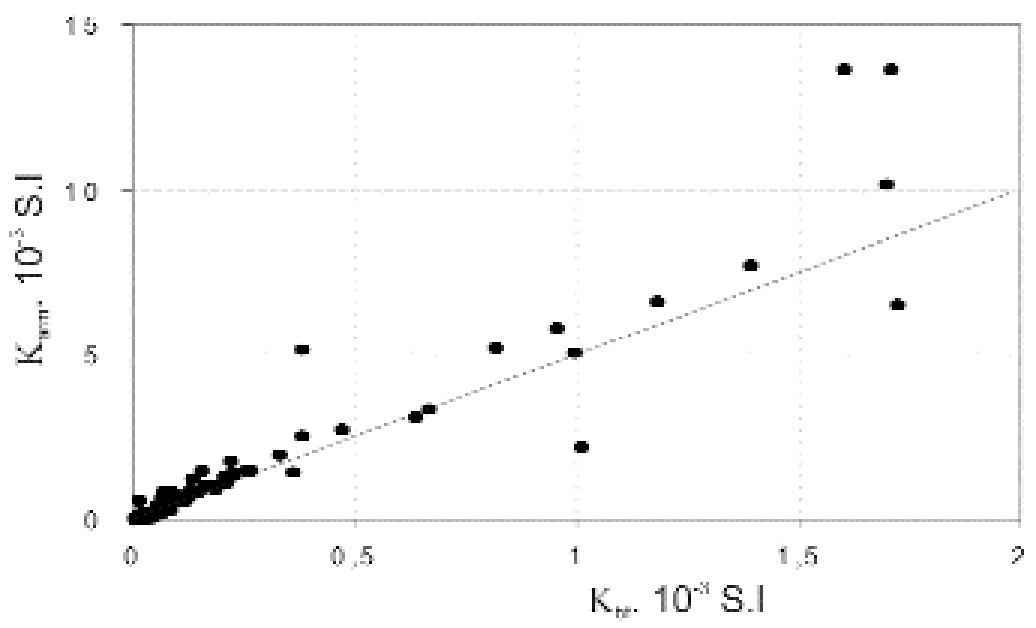

Figure 10 - Diagramme de King (King et al. 1982) de la susceptibilité magnétique anhystérétique (karm $\left.{ }^{*} 10-3\right)$ en fonction de la susceptibilité magnétique volumique (kbf 10-3).

Figure 10 - Relationship between ARM susceptibility (Karm* 10-3) and low-field magnetic susceptibility (103) (King et al. 1982). 
dans les ensembles magnétostratigraphiques dominés par les éboulis se localisent dans la partie inférieure gauche du diagramme et contiennent une très faible concentration en grains magnétiques (moins de à $0.001 \%$ ).

La seconde, qui représente $k_{a m}$ en fonction de $\kappa_{b f}$ (fig. 10), montre que les grains de petite taille donnent de hautes valeurs en $\mathrm{k}_{\mathrm{am}}$, et ce, parce qu'ils sont très efficaces pendant l'acquisition de la rémanence (Maher 1988 ; Dunlop 1995). Cependant, la susceptibilité magnétique permet uniquement d'estimer la concentration en grains magnétiques. Le diagramme montre que la quasi-totalité des échantillons prélevés dans les niveaux archéologiques riches en matériel brûlé se localisent dans la zone caractérisant des grains de magnétite de taille fine. Les échantillons prélevés dans les éboulis montrent de faibles valeurs des deux rapports.

Terminons cet exposé des résultats en insistant sur certains points importants. Cette étude nous a permis d'une part d'identifier des ensembles magnétostratigraphiques, et d'autre part de connaître la nature, la taille et les propriétés des grains magnétiques. Elle a permis également d'avancer des hypothèses en termes de paléoenvironnements.

Toutefois, il faut reconnaître les limites de la méthode, notamment lorsque les sédiments sont très pauvres en oxydes de fer (soit à cause de problèmes de dilution ou de migration du fer, soit du fait d'une roche mère elle-même très pauvre en fer).

Par ailleurs, bien que dans la majorité des cas les éléments formés sous un climat humide présentent de fortes valeurs des différents paramètres magnétiques, il arrive parfois que certains éléments (planchers stalagmitiques, matière diamagnétique) présentent de très faibles valeurs, ce qui peut fausser les interprétations paléoclimatiques.

Pédogenèse ou foyer ? II est important de clarifier les choses. D'une façon générale, les valeurs de la susceptibilité magnétique augmentent dans les sédiments exposés à la pédogenèse, dans les foyers et dans les sédiments qui leur sont proches. Cependant, Ellwood et al. (1995, 1996, 2001), à travers l'étude de plusieurs sites préhistoriques dans le sud-ouest de l'Europe, ont signalé que l'effet des foyers était minime sur les valeurs de la susceptibilité magnétique. Ils affirment par conséquent que la pédogenèse contrôle la majeure partie du signal magnétique. Woodward et al. 1992 montrent que les sédiments non protégés des agents atmosphériques (comme dans les abris-sous-roche et les sites en plein air) ne donnent pas de bons résultats en termes d'interprétation paléoclimatique. Dans le cas de l'Abri Pataud, nous signalons que:

- les niveaux d'éboulis, contemporains d'un climat froid et sec, pauvres en minéraux magnétiques, sont majoritaires ; - il n'y a pas de trace de pédogenèse, excepté dans la partie supérieure du remplissage (niveau archéologique 1, Solutréen) et dans la partie médiane (ensemble magnétostratigraphique 4), qui ont subi une très faible pédogenèse (valeurs de $\chi_{\mathrm{fd}}$ supérieures à $7 \%$, fig. 4B) ;

- les fortes valeurs des différents paramètres magnétiques correspondent aux foyers et à leurs zones de vidange, la nature des oxydes de fer présents dans les foyers étant fonction du nombre de chauffe et de leur intensité, de la nature des grains magnétiques primaires présents et de la température atteinte dans les foyers ;

- dans les zones de prélèvements, il semble n'y avoir eu ni lessivage, ni migrations des éléments fins sur les plans vertical et latéral (les zones correspondant aux foyers sont limitées, et les fortes valeurs concernent uniquement ces foyers). Cette constatation, confirmée par le fait que les grains de taille SP de magnétite et de maghémite (facilement transportables par les eaux interstitielles) se concentrent uniquement dans les foyers et leurs zones de vidange, est toutefois en contradiction avec les interprétations de Farrand (1995) et Agsous et al. (2007). Une discussion plus précise avec les autres approches (essentiellement géoarchéologique) permettrait peut-être de lever cette contradiction.

Les conditions climatiques qui régnaient pendant la mise en place du remplissage (froid et sec) ne sont pas favorables au développement d'une pédogenèse (humidité, température, activité de micro-organismes et présence de matière organique), la susceptibilité magnétique des sédiments de l'abri Pataud est donc contrôlée soit par les grains magnétiques formés dans les foyers lors de la combustion (magnétite et maghémite), soit par les minéraux diamagnétiques ou antiferromagnétiques présents dans les niveaux à éboulis.

\section{CONCLUSION}

Le site de l'abri Pataud est caractérisé par la succession de plusieurs niveaux archéologiques (datés de l'Aurignacien jusqu'au Solutréen), séparés par des niveaux d'éboulis stériles, formés sous un climat globalement froid entre -35000 et -20000 ans (stades isotopiques 2 et 3). Les foyers sont très abondants dans les niveaux d'habitats.

Il est important de noter que le remplissage de ce site présente un enchevêtrement de blocs, cailloux et sédiments plus fins, entrecoupé par des foyers et des nappes de vestiges caractérisant les niveaux dits archéologiques.

Notre étude magnétique a cependant permis de détecter plusieurs ensembles magnétostratigraphiques, trois caractérisés par de fortes valeurs des paramètres magnétiques (EMS 2, 4 et 6 ) et trois autres par de faibles valeurs (EMS 1, 3 et 5) :

- Les premiers (fortes valeurs), correspondant aux niveaux archéologiques sablo-limoneux, riches en nombreux éléments chauffés, présentent un fort pourcentage en grains magnétiques d'origine secondaire (magnétite et maghémite), de taille fine, issus de la transformation des minéraux primaires sous l'effet de la température. Les oxydes de fer présents dans les ensembles magnétostratigraphiques 2 et 4 ne pouvant pas être formés à la suite de phénomènes de pédogenèse, ils sont donc forcément issus de phénomènes de combustion. En effet des oxydes de fer (magnétite) sont formés par réduction de la matière paramagnétique (sidérite) suite à l'augmentation de la température dans les foyers (> à $800 \mathrm{C}^{\circ}$ ). La maghémite est ensuite issue par oxydation de la magnétite (Maher 1986). L'accroissement de la température peut également former 
la maghémite (déshydratation de la lépidocrocite) sans passer par la magnétite (Mullins 1977 ; Guo et al. 2001). L'ensemble magnétostratigraphique 6 occupe la partie supérieure du remplissage, riche en matière organique (traces évidentes de pédogenèse : racines, couleur brune, etc.) et contient de nombreux éléments chauffés (charbons, silex et os brûlés, etc.). Les fortes valeurs de $\chi_{b f}$ et de l'ARI ${ }_{S}$ dans ce niveau sont dues en grande partie au fort pourcentage de grains de maghémite $(\gamma$-Fe2O3) et de magnétite de taille très fine $(\mathrm{SP}<20-25 \mathrm{~nm})$. Ces particules ultrafines se forment pendant la pédogenèse (Zhou et al. 1990 ; Verosub et al. 1993 ; Heller et Evans 1995 ; Deng et al. 2000 ; Liu et al. 2004a).

Par ailleurs, la présence de ces minéraux secondaires est attestée par le faible champ coercitif de ces grains, leur saturation rapide et les fortes valeurs de susceptibilité ferromagnétique.

- Les seconds niveaux (faibles valeurs) concernent les niveaux d'éboulis, formés sur place par l'altération mécanique des cailloux et blocs tombés de la paroi calcaire, mêlés à des sables éoliens. Tous ces éléments se sont formés sous un climat froid et sec. Les grains magnétiques sont d'origine détritique, et sont essentiellement formés par des grains de sidérite (matière paramagnétique), d'hématite (matière antiferromagnétique), et de goethite. Cette fois-ci, les faibles valeurs de $\chi_{b f}$, de l'ARI ${ }_{S}$ et de l'ARA, les fortes valeurs du champ coercitif, la non-saturation des grains (avec champ magnétique fort) et la contribution importante des grains para-, dia- et antiferromagnétiques prouvent véritablement l'origine détritique des grains.

\section{Remerciements}

Nous remercions le Professeur Rochette (CEREGE, Université d'Aix-Marseille) et le Professeur Wagner (Université de Genève) de nous avoir permis d'utiliser une partie de leurs équipements de paléomagnétisme afin d'effectuer nos mesures. Merci également à Messieurs Texier, Lenoble et Bertran d'avoir relu et corrigé ce travail et de nous avoir ainsi permis de réaliser des améliorations significatives. N'oublions pas enfin Madame Djerrab, pour sa patiente contribution, et sans qui ce travail n'aurait pas été possible.

\section{BIBLIOGRAPHIE}

AGSOUS S., LENOBLE A., NESPOULET R., 2007 - L'Abri Pataud, in Texier J.-P. (dir.), Livret-guide de l'excursion de l'Association française de Sédimentologie "Sites préhistoriques du Périgord". Edition numérique 2006, p. 31-43. http://www.pole-prehistoire.com.

BAILEY M.E. et DUNLOP D.J. 1983 - Altemating field characteristics of pseudo-single-domain $(2-14 \mu \mathrm{m})$ and multidomain magnetite. Earth and Planetary Science Letters, 1983, 63, p. 335-352.
BANERJEE S.K., KING J. et MARVIN J. 1981 - A rapid method for magnetic granulometry with application to environmental studies. Geophysical Research Letters, 1981, 8(4), p. 333-336.

BLOEMENDAL J., KING J.W., HALL F.R. et DOH S.-H. 1992 - Rock magnetism of Late Neogene and Pleistocene deep-sea sediments: Relationship to sediment source, diagenetic processes, and sediment lithology. Joumal of Geophysical Research, 1992, 97, p. 4361-4375.

BRICKER H.M. et MELLARS P.A. 1987 - Datation 14C de l'abri Pataud (Les Eyzies, Dordogne) par le procédé “ Accélérateur-Spectromètre de Masse ". L'Anthropologie, 1987, 91(1), p. 227-234.

CHIOTTI L. et NESPOULET R. 2007 - L'apport méthodologique des fouilles de Hallam $L$. Movius à l'abri Pataud (les Eyzies-de-Tayac, Dordogne), Un siècle de construction du discours scientifique en préhistoire. XXVlème Congrès préhistorique de France, Avignon, 21-25 septembre 2004, Société préhistorique française, vol. I, p. 185-195.

COLLOMBAT H. 1993 - Etude des propriétés des sédiments non consolidés : anisotropie et erreurs d'inclinaison paléomagnétique. Thèse de doctorat, Université J. Fourier, Grenoble I, 214 p.

DAY R., FULLER M. et SCHMIDT V.A. 1977 - Hysteresis properties of titanomagnetites: grain size and compositional dependence. Earth and Planetary Science Letters, 1977, 13, p. 260-267.

DEARING, J. A. et FLOWER, R. J. 1982 - The magnetic susceptibility of sedimenting material trapped in Lough Neagh, Northem Ireland and its erosional significance. Limnology and Oceanography, 1982, 17, p. 969-975.

DEKKERS M.J. 1988 - Magnetic properties of natural pyrrhotite part 1: behaviour of initial susceptibility and saturation-magnetization-related rock-magnetic parameters in a grain-size dependant framework. Physics of the Earth and Planetary Interiors, 1988, 52, p. 376-393.

DEKKERS M.J. 2003 - Selected room temperature magnetic parameters as fonction of mineralogy, concentration and grain size. Physics and Chemistry of the Earth, 2003,28 , p. $659-667$

DENG C. L., ZHU R. X., VEROSUB K. L., SINGER M. J., et YUAN B. Y. 2000 - Paleoclimatic significance of the temperature-dependent susceptibility of Holocene loess along a NW-SE transect in the Chinese loess plateau. Geophys. Res. Lett., 2000, 27, p. 3715-3718.

DJERRAB A. 2001 - Etude des propriétés magnétiques du remplissage de sites préhistoriques. Contribution à l'étude stratigraphique de leurs dépôts et à leur évolution géochimique post-dépositionnelle. Thèse de doctorat de l'Université de Perpignan, 667 p. 
DUNLOP D.J. 1986 - Hysteresis properties of magnetite and their dependence on particle size: Attest of pseudosingle domain remanence models. Journal of Geophysical Researc $h, 1986,91$, p. 9569-9584.

DUNLOP D.J. 1995 - Magnetism in rocks (invited American Geophysical Union 75th anniversary paper). Journal of Geophysical Research, 1995, 100, p. 2161-2174.

DUNLOP D.J. 2002 - Theory and application of the Day plot ( $\mathrm{M}_{\mathrm{rs}} / \mathrm{M}_{\mathrm{s}}$ vs. $\left.\mathrm{H}_{\mathrm{Cr}} / \mathrm{H}_{\mathrm{C}}\right)$ 2. Application to data for rocks, sediments and soils. Journal of Geophysical Research, 2002, 107 (B3), EPM 5-1 to 5-15. doi: 10.1029/ 2001JB000487.

ELLWOOD B.B., BALSAM W., BURKART B., LONG G.J. et BUHL M.L. 1986 - Anomalous magnetic properties in rocks containing the mineral siderite: Paleomagnetic implications. Journal of Geophysical Research, 1986, 91, B12, p. $12779-12790$.

ELLWOOD B.B., PETER D.E., BALSAM W. et SCHIEBER J., 1995 - Magnetic and Geochemical Variations as Indicators of Paleoclimate and Archaeological Site Evolution: Examples from 41TR68, Fort Worth, Texas. Journal of Archaeological Science, 1995, 22, p. 409-415.

ELLWOOD B.B., PETRUSO K.M. et HARROLD F.B. 1996 - The Utility of Magnetic Susceptibility for Detecting Paleoclimatic Trends and as a Stratigraphic Comelation Tool: An Example from Konispol Cave Sediments, SW Albania. Journal of Field Archaeology, 1996, 23, p. 263-271.

ELLWOOD B.B., HARROLD F.B., BENOIST S.L., STRAUS L.G., MORALES M. G., PETRUSO K., BICHO N.F., ZILHAO J. et SOLER N. 2001 - Paleoclimate and intersite correlations from Late Pleistocene/Holocene cave sites: results from southern Europe. Geoarcheology: an international journal, 2001, 16(4), p. 433-463.

FARRAND W.R. 1975 - Analysis of the Abri Pataud sediments, in MOVIUS H.L. Jr., Excavation of the Abri Pataud, Les Eyzies (Dordogne). Contributors, American School of Prehistoric Research, Peabody Museum, Harvard University, Cambridge, Massachusetts, bull. $N^{\circ}$ 30, p. 27-68.

FARRAND W.R. 1995 - Etude sédimentologique du remplissage de l'abri Pataud, in BRICKER H.M., Le Paléolithique supérieur de l'abri Pataud. Les fouilles de H.L. Movius, Paris, Maison des Sciences de l'Homme, Documents d'archéologie française, 50, p. 31-65.

FELLAG H. 1996 - Contribution à l'étude des paléoenvironnements et des paléoclimats de la fin du Pléistocène moyen et du Pléistocène supérieur du Sud-Ouest de la France. Analyse pollinique des remplissages des grottes de Suard, Bourgeois-Delaunay, Fontéchevade (Charente), et de l'abri Pataud (Dordogne). Thèse de doctorat, Muséum national d'Histoire naturelle, Paris, $216 \mathrm{p}$.
FELLAG H. 1998 - Apport de l'analyse pollinique à la connaissance du paléoenvironnement du Paléolithique supérieur de l'abri Pataud (Dordogne, France). Bulletin de la Société préhistorique française, 1998, 95(2), p. 171-181.

FORSTER T.H., EVANS M.E. et HELLER F., 1994 - The frequency dependence of low field susceptibility in loess sediments. Geophysical Journal Intemational, 1994, 118, p. 636-642.

GROGAN K. L., GILKES R. J. et LOTTERMOSER B.G. 2003 - Maghemite formation in burnt plant litter at East Trinity, North Queensland, Australia. Clays and Clay Minerals, 2003, 51(4), p. 390-396.

GUO B., ZHU R., BAI L. et FLORINDO F. 2001 - Rock magnetic properties of a loess-paleosol couple along an $\mathrm{N}-\mathrm{S}$ transect in the Chinese Loess Plateau. Science In China (Series D), 2001, 44(12), p. 1099-1109.

HARTSTRA R.L. 1982 - Grain-size dependance of initial susceptibility and saturation magnetization-related parameters of four natural magnetites in the PSD-MD range. Geophys. J. R. Astr. Soc., 1982, 71, p. 477-495.

HEDLEY I.G. 1968 - Chemical remanent magnetization of the $\mathrm{FeOOH}, \mathrm{Fe}_{2} \mathrm{O}_{3}$ system. Physics of the Earth and Planetary Interiors, 1968, 1, p. 103-121.

HEIDER F., ZITZELSBERGER $A$. et FABIAN $K$ 1996 - Magnetic susceptibility and remanent coercive force in grown magnetic crystals from $0.1 \mathrm{~mm}$ to $6 \mathrm{~mm}$. Physics of the Earth and Planetary Interiors, 1996, 93, p. 239-256.

HELLER F. 1978 - Rockmagnetic studies of upper Jurassic limestones from southern Germany. J. Geophys., 1978, 44, p. $525-543$.

HELLER F. et LIU T.S. 1982 - Magnetostratigraphical dating of loess deposits in China. Nature, 1982, 300, p. 431-433.

HELLER F. et EVANS M.E. 1995 - Loess magnetism. Rev. Geophys., 1995, 33, p. 211-240.

HUNT C.P., SINGER M.J., KLETETSCHKA G., TEN PAS J. et VEROSUB K.L. 1995 - Effect of citrate bicarbonate-dithionite treatment on fine-grained magnetite and maghemite. Earth and Planetary Science Letters, 1995,130 , p. $87-94$.

KING J., BANERJEE S.K., MARVIN J. et ÖZDEMIR O. 1982 - A comparison of different magnetic methods for detemining the relative grain size of magnetite in naturel materials : some results from lake sediments. Earth and Planetary Science Letters, 1982, 59, p. 404-419.

LANCI L., HIRT A.M., LOWRIE W., LOTTER A.F., LEMCKE G. et STURM M. 1999 - Mineral-magnetic record of late quaternary climatic changes in a high Alpine lake. Earth and Planetary Science Letters, 1999, 170, p. 49-59. 
LE BORGNE, E., 1955 - Susceptibilité magnétique anormale du sol superficiel. Ann. Geophys., 1955, 11, p. 399-419.

LE BORGNE, E., 1964 - The relationship between the magnetic susceptibility and the history of soils, In : problems in palaeoclimatology. A.E.M, Nairn. Eds. Inter-science. London, p. 666-669.

LE BORGNE E. 1965 - Les propriétés magnétiques du sol. Application à la prospection des sites archéologiques. Archaeophysica, 1965, 1, p. 1-20.

LIU Q.S., BANERJEE S.K., JACKSON M.J., CHEN F.H., PAN Y.X. et ZHU R.X. 2004a - Detemining the climatic boundary between the Chinese loess and palaeosol: Evidence from aeolian coarse-grained magnetite. Geophys. J. Int., 2004, 156, p. 267-274.

LIU Q., JACKSON M.J., YU Y., CHEN F., DENG C. et ZHU R. 2004b - Grain size distribution of pedogenic magnetic particles in Chinese loess//paleosols. Geophysical Research Letters, 2004, 31, L22603, doi:10.1029/2004GL021090.

LOWRIE W. et HELLER F. 1982 - Magnetic properties of marine limestones. Rev. Geophys., 1982, 20, p. 171-192.

LOWRIE W. 1990 - Identification of ferromagnetic minerals in a rock by coercivity and unblocking temperature properties. Geophysical Research Letters, 1990, 17(2), p. 159-162.

MAHER B.A. 1986 - Characterisation of soil by mineral magnetic measurements. Physics of the Earth and Planetary Interiors, 1986, 42, p. 76-92.

MAHER B.A. 1988 - Magnetic properties of some synthetic submicron magnetites. Geophys. J., 1988, 94, p. 83-96.

MAHER B.A. et THOMPSON R. 1992 - Paleoclimatic significance of the mineral magnetic record of the Chinese loess and paleosols. Quaternary Research, 1992, 37, p. 155-170.

MOONEY S.D., GEISS C. et SMITH M.A. 2002 - The use of mineral magnetic parameters to characterize archaeological ochres. Journal of Archaeological Science, 2002, 29, doi:10.1006/ jasc.

MOVIUS H.L. 1963 - L'Age du Périgordien, de l'Aurignacien et du Protomagdalénien en France sur la base des datations au Carbonne 14. In : Aurignac et l'Aurignacien : Centenaire des fouilles d'Edouard Lartet, Bulletin de la Société méridionale de spéléologie et de préhistoire, Toulouse, tomes VI-IX, 1956-1959, p. 130-142.

MOVIUS H.L. 1971 - Radiocarbon dating of the upper Palaeolithic sequence at the Abri Pataud, Les Eyzies, Dordogne. In The Origin of Homo Sapiens (Ecology and Conservation), 3, UNESCO, p. 253-260.

MOVIUS H.L. (dir) 1975 - Excavations of the Abri Pataud, Les Eyzies (Dordogne) Contributors. American school of prehistoric research. Peabody Museum of Archaeology and Ethnologie. Harvard University, Bull. 30, 305 p.

MOVIUS H. L. (dir.) 1977 - Excavation of the Abri Pataud, Les Eyzies, (Dordogne). Stratigraphy. American School of P rehistoric Research, Peabody Museum, Harvard University, Cambridge, Massachusetts, bull. 31, 167 p.

MULLINS C.E. 1977 - Magnetic susceptibility of the soil and its significance in soil science - a review. J. Soil Sci., 1977,28 , p. 223-246.

NESPOULET R. et CHIOTTI L. 2007a - L'occupation humaine de l'abri Pataud il y a 22000 ans (Les Eyzies-deTayac, Dordogne). Direction régionale des affaires culturelles Aquitaine, Service régional de l'archéologie Bilan scientifique régional 2005, p. 35-37.

NESPOULET R. et CHIOTTI L. 2007b - 1953-2004 : la collection Movius de l'abri Pataud (les Eyzies-de-Tayac, Dordogne). In : Un siècle de construction du discours scientifique en préhistoire, XXVlème Congrès préhistorique de France, Avignon, 21-25 septembre 2004, Société préhistorique française, vol. II, p. 185-196.

ÖZDEMIR O. et BANERJEE S.K. 1982 - A preliminary magnetic study of soil samples from west-central Minnesota. Earth and Planetary Science Letters, 1982, 59, p. 393-403.

ÖZDEMIR O. et DUNLOP D.J. 1996 - Thermoremanence and Néel temperature of goethite. Geophysical Research Letters, 1996, 23, p. 921-924.

PARRY L.G. 1965 - Magnetic properties of dispersed magnetite powders. Philosophical Magazine, 1965, 11(110), p. 303-312.

PARRY L.G. 1981 - The influence of fine structures on the remanence of multidomain particles of magnetite and titanomagnetite. Physics of the Earth and Planetary Interiors, 1981, 26, p. 63-71.

PETERS C. et DEKKERS M.J. 2003 - Selected room temperature magnetic parameters as a function of mineralogy, concentration and grain size. Physics and Chemistry of the Earth, 2003, 28, p. 659-667.

PRZEPIERA K. et PRZEPIERA A 2003 - Thermal transformations of selected transition metals oxyhydroxides. J. Therm. Anal. Cal., 2003, 74, p. 659-666.

RAHMAN A.A., DUNCAN A.D. et PARRY L.G. 1973 - Magnetization of multidomain magnetite particles. Rivista Italiana di Geofisica, 1973, 22 (5/6), p. 259-266.

ROBERTS A.P., CUI Y.-L. et VEROSUB K.L. 1995 - Wasp-waisted hysteresis loops: mineral magnetic characteristics and discrimination of components in mixed magnetic systems, Journal of Geophysical Research, 1995, 100, 17909-17924. 
ROBINSON S.G. 1986 - The late Pleistocene paleoclimatic record of North Atlantic deep-sea sediments revealed by mineral magnetic measurements. Physics of the Earth and Planetary Interiors, 1986, 42, p. 22-47.

ROCHEITE P. et FILLION, G. 1989 - Field and temperature behavior of remanence in synthetic goethite : paleomagnetic implications. Geophysical Research Letters, 1989, 16 , p. $851-854$.

RUAN H. D., FROST R.L. et KLOPROGGE J.T. 2001 - The behavior of hydroxyl units of synthetic goethite and its dehydroxylated product hematite. Spectrochim. Acta, A, 2001, 57, p. 2575-2586.

RUMMERY T.A., BLOEMENDAL J., DEARING J. et OLDFIELD F. 1979 - The persistence of fire-induced magnetic oxides in soils and lake sediments. Ann. Geophys., 1979, 35 , p. 103-107.

RUMMERY T. A. 1983 - The use of magnetic measurements in interpreting the fire histories of lake basins, Hydrobiologia,1983, 103, p. 53-58.

STOCKHAUSEN $H$. et ZOLITSCHKA B. 1999 Environmental changes since 13,000 cal. BP reflected in magnetic and sedimentological properties of sediments from Lake Holzmaar (Germany). Quaternary Science Reviews, 1999, 18, p. 913-925.

THOMPSON R., BATTARBEE R.W., O'SULLIVAN P.E. et OLDFIELD F. 1975 - Magnetic susceptibility of lake sediments. Limnology and Oceanography, 1975, 20, p. 687-698.
THOMPSON R. et OLDFIELD F. 1986 - Environmental Magnetism. Allen and Unwin, London, $227 \mathrm{p}$.

VEROSUB K.L., FINE P., SINGER M.J. et TENPAS J. 1993 - Pedogenesis and paleoclimate: Interpretation of the magnetic susceptibility record of Chinese loess-paleosol sequences. Geology, 1993, 21, p. 1011-1014.

VOGEL J.C. et WATERBOLK H.T. 1963 - Groningen radiocarbon dates, IV, American Journal of Science, 1963, 5, p. 163-202.

VOGEL J.C. et WATERBOLK H.T. 1967 - Groningen radiocarbon dates, VII, American Journal of Science, 1967, 9, p. 107-155.

VOGEL J.C. et WATERBOLK H.T. 1972 - Groningen radiocarbon dates X, American Journal of Science, 1972,14 , p. 6-110.

WESTPHAL M., GUREVITCH E.L., SAMSONOV B.V., FEINBERG H. et POZZI J.P. 1998 - Magnetostratigraphy of the lower Triassic volcanics from deep drill SG6 in western Siberia: evidence for long-lasting Permo-Triassic volcanic activity. Geophysical Journal International, 134, p. 254-266.

WOODWARD J.C, LEWIN J., et MACKLIN M.G. 1992 Alluvial sediment sources in a glaciated catchment; the Voidomatis Basin, northwest Greece, Earth Surface Processes and Landforms, 17, p. 205-216.

ZHOU L.P., OLDFIELD F., WINTLE A.G., ROBINSON S.G., et WANG J.D. 1990 - Partly pedogenic origin of magnetic variations in Chinese loess. Nature, 1990, 346, p. 737-739. 